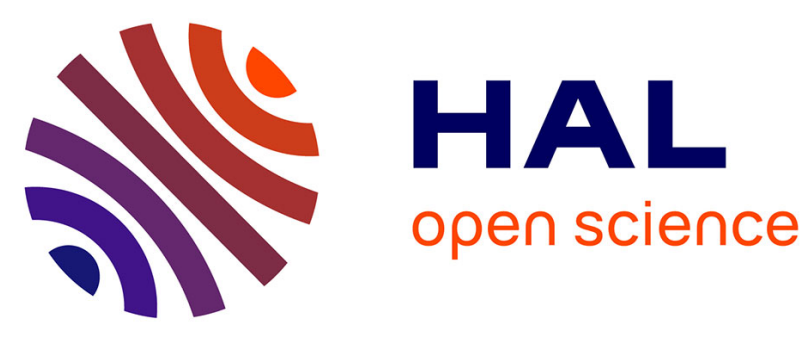

\title{
Matched asymptotic expansion method for an homogenized interface model
}

Giuseppe Geymonat, Sofiane Hendili, Francoise Krasucki, Marina Vidrascu

\section{To cite this version:}

Giuseppe Geymonat, Sofiane Hendili, Francoise Krasucki, Marina Vidrascu. Matched asymptotic expansion method for an homogenized interface model. Mathematical Models and Methods in Applied Sciences, 2014, 24 (3), pp.573-597. 10.1142/S0218202513500607 . hal-00757005v2

\section{HAL Id: hal-00757005 \\ https://hal.science/hal-00757005v2}

Submitted on 4 Mar 2015

HAL is a multi-disciplinary open access archive for the deposit and dissemination of scientific research documents, whether they are published or not. The documents may come from teaching and research institutions in France or abroad, or from public or private research centers.
L'archive ouverte pluridisciplinaire HAL, est destinée au dépôt et à la diffusion de documents scientifiques de niveau recherche, publiés ou non, émanant des établissements d'enseignement et de recherche français ou étrangers, des laboratoires publics ou privés. 


\title{
MATCHED ASYMPTOTIC EXPANSION METHOD FOR AN HOMOGENIZED INTERFACE MODEL
}

\author{
GIUSEPPE GEYMONAT \\ Laboratoire de Mécanique des Solides, UMR-CNRS 7649, \\ Ecole Polytechnique, Route de Saclay, 91128 Palaiseau Cedex, France \\ giuseppe.geymonat@lms.polytechnique.fr \\ SOFIANE HENDILI \\ CEA, DEN, DANS, DM2S, SEMT, \\ Laboratoire de Modélisation et de Simulation des Structures \\ 91191 Gif-sur-Yvette, France. \\ s_hendili@yahoo.fr \\ FRANÇOISE KRASUCKI* \\ I3M, UMR-CNRS 5149 \\ Université de Montpellier II, Place Eugène Bataillon, 34095 Montpellier Cedex 5, France \\ krasucki@math.univ-montp2.fr \\ MARINA VIDRASCU \\ EPI REO, INRIA Rocquencourt, \\ BP 10578153 Le Chesnay Cedex, France \\ and Laboratoire Jacques-Louis Lions, UMR-CNRS 7958 \\ Université Pierre et Marie Curie, Boîte courrier 187, 75252 Paris Cedex 05, France \\ marina.vidrascu@inria.fr \\ Received (Day Month Year) \\ Revised (Day Month Year) \\ Communicated by (xxxxxxxxxx)
}

\begin{abstract}
Our aim is to demonstrate the effectiveness of the matched asymptotic expansion method in obtaining a simplified model for the influence of small identical heterogeneities periodically distributed on an internal surface on the overall response of a linearly elastic body. The results of several numerical experiments corroborate the precise identification of the different steps, in particular of the outer/inner regions with their normalized coordinate systems and the scale separation, leading to the model.
\end{abstract}

Keywords: Multi-materials; multi-scaling; asymptotic analysis, elasticity, numerical experiments.

AMS Subject Classification: 35C20, 35J20, 74A40, 74B05, 74G10, 74G15, 74Q05

${ }^{*}$ Corresponding author. 


\section{Introduction}

The study of boundary value problems where the domain and/or the coefficients have singularities has attracted the interest of many researchers of the mathematical, of the physical and of the engineering communities. Hence the corresponding literature is huge. Roughly speaking one can distinguish problems and methods whose results have been fully justified by rigorous mathematical methods from problems and methods whose results are mainly still formal. This distinction is not definitive since the history of the subject indicates that problems and methods can change of status with the development of new mathematical tools. This has been the case e.g. for the problems connected with homogenisation, with the behaviour of the solutions near singular points of the boundary, with the perturbation of the coefficients giving rise to ill-posed problems. In the case of the matched asymptotic expansion method there are situations where it can be fully mathematically justified, see e.g. Ref. 15, 16. The aim of this paper is to obtain simplified models of the influence on the overall response of a linearly elastic body of small identical heterogeneities periodically distributed on an internal surface $\omega$ (cf. figure 1). Let us strength that the successful classical methods of the homogenisation the-

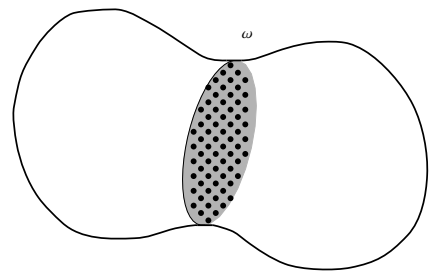

Fig. 1. The structure with the layer of heterogeneities

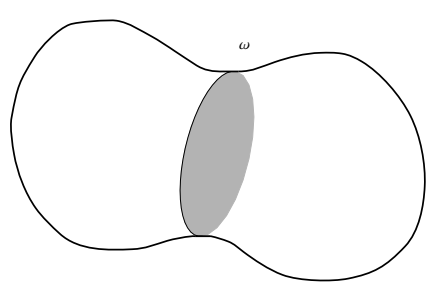

Fig. 2. The structure without the layer of heterogeneities

ory used when the heterogeneities are distributed in a volume, to obtain suitably equivalent macroscopic problems cannot be applied in the present situation since the heterogeneities are only localized near a surface. The goal (and the difficulty) of the analysis is to approximate the initial problem with a set of new ones where the layer of heterogeneities is replaced by a surface (cf. figure 2) on which particular jumping conditions are defined. These are the macroscopic problems. Following a formal method used by Nguetseng \& Sanchez-Palencia ${ }^{21}$ for heterogeneities located near the boundary, the influence of the heterogeneities will be obtained trough the formal analysis of an associated internal boundary layer effect. The analysis of this internal boundary layer uses the matched asymptotic expansion method and gives rise to the macroscopic problems. This method has been recently developed for other kind of heterogeneities localized near an internal surface in e.g. Ref. 1, 19 and 8. Our aim in the present paper is to give a precise description of the matched asymptotic expansion method for the situation considered here and to prove its 
effectiveness through suitable numerical experiments. However we will not analyse here the numerical methods used to realize the numerical experiments; this analysis is done in Ref. 10.

In section 2 we give the precise statement of the problem in the unknowns equilibrium displacement vector field $\boldsymbol{u}^{\varepsilon}$ and equilibrium stress tensor field $\boldsymbol{\sigma}^{\varepsilon}$ of the structure. In section 3 we present the steps of the inner-outer matched asymptotic expansion method. These steps are:

(1) definition for every $\varepsilon>0$ of the inner region $\Omega^{i n, \varepsilon}$ near the layer of the heterogeneities and of the outer region $\Omega^{\text {out }, \varepsilon}$ far from the heterogeneities with a non-empty matching region $\Omega^{m, \varepsilon}:=\Omega^{\text {out }, \varepsilon} \cap \Omega^{\text {in, } \varepsilon} \neq \emptyset$;

(2) introduction of suitable normalized system of coordinates in each region in such a way that for $\varepsilon \rightarrow 0$ the outer region and the inner region converge to fixed domains: the inner and the outer domain;

(3) use of an Ansatz to a priori introduce an asymptotic expansion for the unknown $\boldsymbol{u}^{\varepsilon}$ in the inner and in the outer domain;

(4) deduction from the elasticity stress-strain law of an asymptotic expansion of $\boldsymbol{\sigma}^{\varepsilon}$ in the inner and in the outer domain;

(5) deduction of the boundary value problems for each term of the asymptotic expansion in the inner and in the outer domain;

(6) introduction of the matching conditions in order to obtain well-posed boundary value problems.

In Section 4 we study the first two order outer and inner approximations. In particular we prove that the zero-order outer and inner approximations do not see the heterogeneities. This is due to the particular choice of the geometry and of the material characteristics of the heterogeneities. In the first order outer approximation the layer of heterogeneities is replaced by a non-homogeneous transmission condition on the interface $\omega$. The left members of the transmission conditions depend linearly on the zero-order outer approximation with coefficients depending on the type of heterogeneity. A remarkable characteristic of these coefficients is that, as in homogenisation, they can be computed once for all on an elementary cell.

In Section 5 we present some numerical results in order to prove the effectiveness of our method in particular when the number of heterogeneities increases. In Section 6 we give some further comments and comparison with some results obtained in the case of an $\varepsilon$-small layer of linearly elastic material.

The convention of sum over repeated indexes will be employed. Furthermore, Latin indexes range in $\{1,2,3\}$ and Greek indexes range in $\{2,3\}$. The $2 \mathrm{D}$ or $3 \mathrm{D}$ measure of a set $A$ will be always denoted by $|A|$. The norm of the elasticity tensor $\boldsymbol{B}=\left(B_{i j k l}\right)$ is $\|\boldsymbol{B}\|$; since the elasticity tensor can be identified with a symmetric matrix one can choose the spectral norm (or the euclidean norm or any other norm since all the norms are equivalent). The spectral norm can be easily expressed in terms of the Kelvin moduli (see e.g. ${ }^{22},{ }^{20}$ ). 


\section{Statement of the problem}

Let $\left(\boldsymbol{e}_{1}, \boldsymbol{e}_{2}, \boldsymbol{e}_{3}\right)$ be the canonical basis of $\mathbb{R}^{3}$ and let $\Omega$ be an open domain of $\mathbb{R}^{3}$ with smooth boundary $\partial \Omega$. Let $\omega$ be the intersection of $\Omega$ with the coordinate plane of equation $x_{1}=0 ; \omega$ is assumed to be a non empty bounded domain in $\mathbb{R}^{2}$ having a positive two-dimensional measure and a smooth boundary. We assume that across $\omega$ small (and all equal) $3 D$ heterogeneities are periodically distributed. (cf. figure $1)$.

More precisely, let $\hat{Y} \subset \mathbb{R}^{2}$ be the basis of a periodic planar net and let $I$ be a non-empty $3 D$ domain contained in $\mathbb{R} \times \hat{Y}$ with smooth boundary $\partial I$, with diameter $d$ satisfying $0<\frac{d}{\operatorname{diam}(\Omega)}<+\infty$ and such that $I \cap\left\{x_{1}=0\right\}$ is strictly contained in $\hat{Y}$. (cf. figure 3 ).
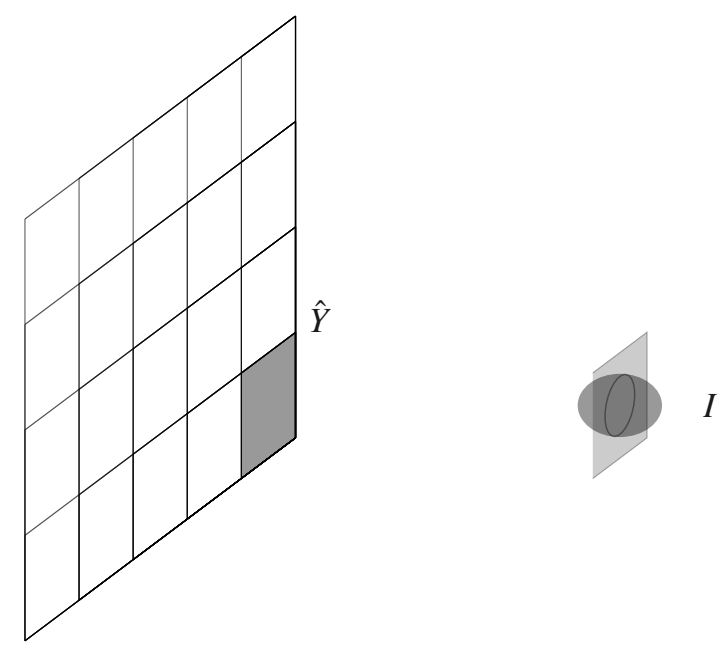

Fig. 3. Representation of $\hat{Y}$ and of $I$

We assume that $\omega$ is contained in the union of $\mathcal{N}(\varepsilon) \approx \frac{\operatorname{aire}(\omega)}{\operatorname{aire}(\hat{Y})} \varepsilon^{-2}$ sets $\varepsilon \hat{Y}$. We also assume that the heterogeneities have a diameter $\varepsilon d$ and fill every domain $\varepsilon I$. We denote with $\varepsilon \partial I$ the boundary of $\varepsilon I$ and with $I^{\varepsilon}$, resp. $\partial I^{\varepsilon}$, the union of all the heterogeneities, resp. of all their boundaries.

Hence from a geometrical point of view there are two natural length scales: the first is a global one (the $3 D$-diameter of $\Omega$ or the $2 D$-diameter of $\omega$ ) the other one is a local one connected with the heterogeneities (e.g. the diameter of each heterogene- 
ity). The ratio between these two scales will be denoted by $\varepsilon$. More precisely, the parameter $\varepsilon$ is a non-dimensional parameter characterizing the geometrical distribution of the heterogeneities in the structure since, at the same time, it characterizes the ratio between the diameter of any heterogeneity $(\varepsilon d)$ and the diameter of $\Omega$ and the ratio between the diameter of the period $(\varepsilon \hat{Y})$ of the planar net and the diameter of the planar set $\omega$ supporting the heterogeneities.

We assume that, outside the heterogeneities, the structure $\Omega$ is constituted by a linearly hyper-elastic material whose rigidity tensor $\boldsymbol{A}$ satisfies the usual assumptions of symmetry and positivity (we also assume for simplicity that $\boldsymbol{A}$ does not depend on $\boldsymbol{x}$ ). As far as the heterogeneities are concerned the following situations can be of interest:

(i) hyper-elastic soft or hard inclusions characterized by a rigidity tensor $\boldsymbol{A}^{\varepsilon, I}$ depending on $\varepsilon$,

(ii) rigid inclusions,

(iii) holes.

In this paper we will consider the case of soft hyper-elastic inclusions, i.e. we assume that

$$
\boldsymbol{A}^{\varepsilon, I}=\varepsilon^{p} \boldsymbol{A}^{I}, \boldsymbol{A}^{I} \simeq \boldsymbol{A}, p \in \mathbb{N}
$$

where $\boldsymbol{A}^{I} \simeq \boldsymbol{A}$ means that $0<\frac{\left\|\boldsymbol{A}^{I}\right\|}{\|\boldsymbol{A}\|}<+\infty$. We also assume that $\boldsymbol{A}^{I}$ satisfies the usual symmetry and positivity assumptions. The case of holes has been considered in a previous paper ${ }^{9}$ (see also ${ }^{19}$ ) and the cases of hard hyper-elastic (resp. rigid) inclusions will be studied in a forthcoming paper.

Thanks to (2.1) the parameter $\varepsilon$ is a non-dimensional parameter characterizing at the same time the geometrical distribution of the heterogeneities in the structure and the ratio between the rigidity of the heterogeneities and the rigidity of the structure. For simplicity we assume that the structure is free of applied body forces. The structure is clamped on a part $\Gamma_{0} \subset \partial \Omega$ (of surface measure $>0$ ) and on the complementary part $\Gamma_{F}$ of the boundary $\partial \Omega$ is submitted to applied surface forces $\boldsymbol{F}$. Obviously one can consider other type of boundary conditions (e.g. a combination of some components of the stress vector and of the displacement).

Let $\boldsymbol{u}^{\varepsilon}$ and $\boldsymbol{\sigma}^{\varepsilon}$ be the equilibrium displacement vector field and the equilibrium stress tensor field of the structure. They are, for every $\varepsilon>0$ fixed, the solutions of 
the following classical elastostatic problem $P^{\varepsilon}$ :

$$
\begin{cases}\boldsymbol{d i v} \boldsymbol{\sigma}^{\varepsilon}=\mathbf{0} & \text { in } \Omega \\ \boldsymbol{\sigma}^{\varepsilon}=\boldsymbol{A} \gamma\left(\boldsymbol{u}^{\varepsilon}\right) & \text { in } \Omega \backslash I^{\varepsilon} \\ \boldsymbol{\sigma}^{\varepsilon}=\varepsilon^{p} \boldsymbol{A}^{I} \gamma\left(\boldsymbol{u}^{\varepsilon}\right) & \text { in } I^{\varepsilon}, p \in \mathbb{N} \\ \boldsymbol{\sigma}^{\varepsilon} \boldsymbol{n}=\boldsymbol{F} & \text { on } \Gamma_{F} \\ \boldsymbol{u}^{\varepsilon}=\mathbf{0} & \text { on } \Gamma_{0}\end{cases}
$$

Notice that on the internal boundaries $\partial I^{\varepsilon}$ the following two continuity conditions (also called transmission conditions) hold:

$$
\begin{cases}{\left[\boldsymbol{u}^{\varepsilon}\right]_{\partial I^{\varepsilon}}=\mathbf{0}} & \text { on } \partial I^{\varepsilon} \\ {\left[\boldsymbol{\sigma}^{\varepsilon} \boldsymbol{n}\right]_{\partial I^{\varepsilon}}=\mathbf{0}} & \text { on } \partial I^{\varepsilon}\end{cases}
$$

where as usual $[\mathbf{w}]_{\partial I^{\varepsilon}}$ denotes the jump of $\mathbf{w}$ across $\partial I^{\varepsilon}$ and $\left[\boldsymbol{\sigma}^{\varepsilon} \boldsymbol{n}\right]_{\partial I^{\varepsilon}}$ denotes the jump of the stress vector. Let us remark that (2.2) has, for every $\varepsilon>0$, a unique solution $\boldsymbol{u}^{\varepsilon} \in \boldsymbol{H}^{1}(\Omega)$.

In order to see the influence of the heterogeneities when $\varepsilon \rightarrow 0$, we suitably adapt the inner-outer matched asymptotic expansion method to obtain an approximation of $\boldsymbol{u}^{\varepsilon}$ and of $\boldsymbol{\sigma}^{\varepsilon}$. A first general presentation of the method has been given in a seminal book by M. Van Dyke ${ }^{25}$ (see also J. Sanchez Hubert \& E. Sanchez Palencia ${ }^{23}$ for a more recent presentation).

\section{The inner-outer matched asymptotic expansion method}

\subsection{Separation of the global or macroscopic scale of the structure from the microscopic scale of the heterogeneities}

In order to separate the macroscopic from the microscopic scale we introduce two zones. The first one, situated far from the heterogeneities, cf. figure 4, is defined by $\Omega^{\text {out }, \varepsilon}:=\left\{\boldsymbol{x} \in \Omega ;\left|x_{1}\right|>\frac{\varepsilon}{2}\right\}=\Omega^{+, \varepsilon} \cup \Omega^{-, \varepsilon}$ where

$$
\Omega^{+, \varepsilon}:=\left\{\boldsymbol{x} \in \Omega ; x_{1}>\frac{\varepsilon}{2}\right\}, \Omega^{-, \varepsilon}:=\left\{\boldsymbol{x} \in \Omega ; x_{1}<-\frac{\varepsilon}{2}\right\}
$$

One can say, roughly speaking, that in this zone the fields $\boldsymbol{u}^{\varepsilon}$ and $\boldsymbol{\sigma}^{\varepsilon}$ do not see the heterogeneities. The second zone, situated near the the heterogeneities, cf. figure 5 , is defined by $\Omega^{i n, \varepsilon}:=\left\{\boldsymbol{x} \in \Omega ;\left|x_{1}\right|<\frac{\eta(\varepsilon)}{2}\right\}$ where $\eta(\varepsilon)$ is a function satisfying the following conditions:

$$
\left\{\begin{array}{l}
\lim _{\varepsilon \rightarrow 0} \eta(\varepsilon)=0 \\
\lim _{\varepsilon \rightarrow 0} \frac{\eta(\varepsilon)}{\varepsilon}=+\infty
\end{array}\right.
$$

In this zone the fields $\boldsymbol{u}^{\varepsilon}$ and $\boldsymbol{\sigma}^{\varepsilon}$ are rapidly oscillating with respect to the variables $\left(x_{2}, x_{3}\right)$. It is important to stress that the two zones have a non-empty intersection, cf. figure $6, \Omega^{m, \varepsilon}:=\left\{\boldsymbol{x} \in \Omega ; \frac{\varepsilon}{2}<\left|x_{1}\right|<\frac{\eta(\varepsilon)}{2}\right\}$. 


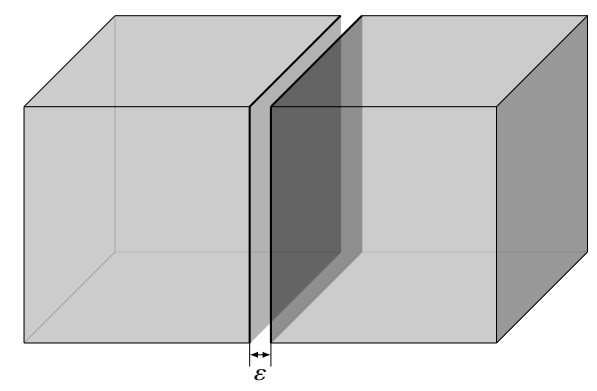

Fig. 4. $\Omega^{\text {out }, \varepsilon}=\Omega^{+, \varepsilon} \cup \Omega^{-, \varepsilon}$

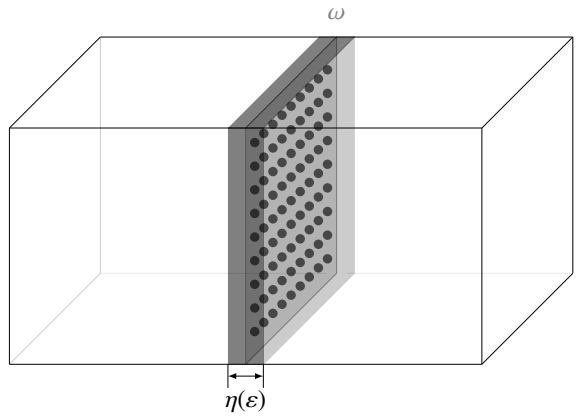

Fig. 5. $\Omega^{i n, \varepsilon}$

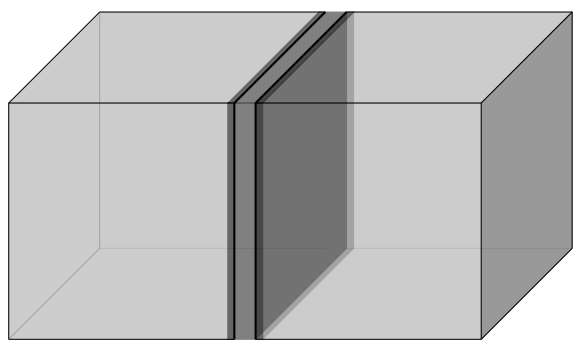

Fig. 6. $\Omega^{m, \varepsilon}$

\subsection{Introduction of normalized coordinate systems}

- When $\varepsilon \rightarrow 0$, the domain $\Omega^{+, \varepsilon} \cup \Omega^{-, \varepsilon}=\left\{\boldsymbol{x} \in \Omega ;\left|x_{1}\right|>\varepsilon\right\}$ tends to a fixed domain $\Omega^{-} \cup \Omega^{+}=\Omega \backslash \omega=\left\{\boldsymbol{x} \in \Omega ;\left|x_{1}\right|>0\right\}$ which is also called the outer domain. The outer system of coordinates is $\boldsymbol{x}:=\left(x_{1}, x_{2}, x_{3}\right) \in \Omega \backslash \omega$.

- Since $\omega$ is contained in the union of $\mathcal{N}(\varepsilon)$ sets $\varepsilon \hat{Y}$, as in the periodic homogenisation procedure the position $\boldsymbol{z}:=\left(z_{1}, z_{2}, z_{3}\right)$ of a point $M$ in the inner domain 
can be given by two independent data:

i) the macroscopic position $(0, \hat{\boldsymbol{x}}):=\left(0, x_{2}, x_{3}\right) \in \omega$ of the center of the period $\varepsilon \hat{Y}$ containing the projection of $M$ on $\omega$

ii) the microscopic position defined as the position of the point $M$ with respect to this center.

In the following, without loss of generality, we take $\hat{Y}=]-\frac{\alpha}{2}, \frac{\alpha}{2}[\times]-\frac{\alpha}{2}, \frac{\alpha}{2}[$.

To obtain a domain and a coordinate system independent from $\varepsilon$, one applies to the inner domain a dilatation of the variables $\varepsilon y_{i}$ of a factor $\frac{1}{\varepsilon}$. Thus, using (3.1) and the periodicity assumption on the heterogeneities, the inner domain is $\omega \times Y$ where $Y:=\mathbb{R} \times \hat{Y}$ is called the basic cell and the relation between the coordinates $\boldsymbol{z}$ of the point $M$ and the point $((0, \hat{\boldsymbol{x}}), \boldsymbol{y})$ of the inner domain is given by:

$$
\boldsymbol{z}=(0, \hat{\boldsymbol{x}})+\varepsilon \boldsymbol{y}
$$

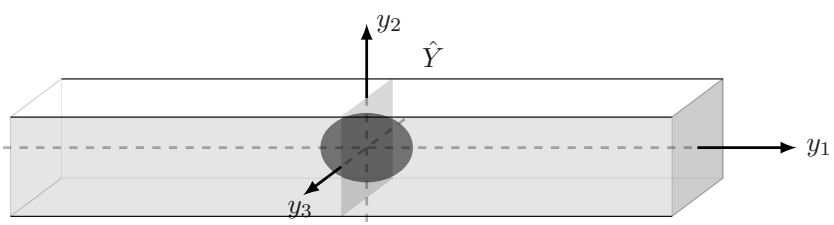

Fig. 7. The elementary cell $Y$

This correspondence implies that the operators divergence $(\boldsymbol{d i v})$ and symmetric gradient $(\gamma)$ must be reformulated in terms of the macroscopic $\hat{\boldsymbol{x}}$ and the microscopic $\boldsymbol{y}=\left(y_{1}, \hat{\boldsymbol{y}}\right)$ coordinates in the following way:

$$
\begin{array}{r}
\boldsymbol{d i v} \boldsymbol{\tau}(\hat{\boldsymbol{x}}, \boldsymbol{y})=\boldsymbol{d i v}_{x} \boldsymbol{\tau}(\hat{\boldsymbol{x}}, \boldsymbol{y})+\frac{1}{\varepsilon} \boldsymbol{d i v}_{y} \boldsymbol{\tau}(\hat{\boldsymbol{x}}, \boldsymbol{y}) \\
\gamma(\boldsymbol{v}(\hat{\boldsymbol{x}}, \boldsymbol{y}))=\gamma_{\boldsymbol{x}}(\boldsymbol{v}(\hat{\boldsymbol{x}}, \boldsymbol{y}))+\frac{1}{\varepsilon} \gamma_{\boldsymbol{y}}(\boldsymbol{v}(\hat{\boldsymbol{x}}, \boldsymbol{y}))
\end{array}
$$

with:

$$
\left\{\begin{array}{l}
\boldsymbol{d i v}_{x} \boldsymbol{\tau}=\frac{\partial \tau_{i j}}{\partial x_{j}} \boldsymbol{e}_{i} \quad, \boldsymbol{d i v}_{y} \boldsymbol{\tau}=\frac{\partial \tau_{i j}}{\partial y_{j}} \boldsymbol{e}_{i} \\
\left(\boldsymbol{\gamma}_{x}(\boldsymbol{v})\right)_{i j}=\frac{1}{2}\left(\frac{\partial v_{j}}{\partial x_{i}}+\frac{\partial v_{i}}{\partial x_{j}}\right),\left(\boldsymbol{\gamma}_{y}(\boldsymbol{v})\right)_{i j}=\frac{1}{2}\left(\frac{\partial v_{j}}{\partial y_{i}}+\frac{\partial v_{i}}{\partial y_{j}}\right)
\end{array}\right.
$$

\subsection{Ansatz: introduction of the two a priori asymptotic} expansions of $\boldsymbol{u}^{\varepsilon}$

- the outer expansion

$$
\boldsymbol{u}^{\varepsilon}\left(x_{1}, x_{2}, x_{3}\right)=\sum_{i=0}^{\infty} \varepsilon^{i} \boldsymbol{u}^{i}\left(x_{1}, x_{2}, x_{3}\right)
$$


- the inner expansion

$$
\boldsymbol{u}^{\varepsilon}\left(x_{1}, x_{2}, x_{3}\right)=\sum_{i=0}^{\infty} \varepsilon^{i} \boldsymbol{v}^{i}\left(\hat{\boldsymbol{x}}, y_{1}, \hat{\boldsymbol{y}}\right)
$$

where the $\boldsymbol{v}^{i}$ are $\hat{Y}$-periodic, i.e. such that:

$$
\boldsymbol{v}^{i}\left(\hat{\boldsymbol{x}}, y_{1}, \hat{\boldsymbol{y}}+p \alpha \boldsymbol{e}_{2}+q \alpha \boldsymbol{e}_{3}\right)=\boldsymbol{v}^{i}\left(\hat{\boldsymbol{x}}, y_{1}, \hat{\boldsymbol{y}}\right) \quad \forall(p, q) \in \mathbb{Z}^{2}
$$

and $\hat{\boldsymbol{x}} \in \omega$ has to be considered as a parameter.

\subsection{Deduction of the outer and inner expansion of $\sigma^{\varepsilon}$}

We use the constitutive equations and the inner and outer expansion of $\boldsymbol{u}^{\varepsilon}$ to determinate the outer and the inner expansion of $\boldsymbol{\sigma}^{\varepsilon}$. Since the material in the inclusions is hyper-elastic and characterized by the hyper-elastic fourth-order tensor $\boldsymbol{A}^{\varepsilon, I}=\varepsilon^{p} \boldsymbol{A}^{I}$ with $\boldsymbol{A}^{I} \simeq \boldsymbol{A}$ and $p \in \mathbb{N}$ and since outside the heterogeneities the structure is constituted by a linearly hyper-elastic material whose rigidity tensor is $\boldsymbol{A}$, the stress tensor field has the following asymptotic expansion:

- Outer expansion:

$$
\boldsymbol{\sigma}^{\varepsilon}(\boldsymbol{x})=\sum_{i=0}^{\infty} \varepsilon^{i} \boldsymbol{\sigma}^{i}(\boldsymbol{x})
$$

with

$$
\boldsymbol{\sigma}^{i}=\boldsymbol{A} \boldsymbol{\gamma}_{x}\left(\boldsymbol{u}^{i}\right)
$$

- Inner expansion:

- in $Y \backslash I$ :

$$
\boldsymbol{\sigma}^{\varepsilon}(\boldsymbol{x})=\sum_{i=-1}^{\infty} \varepsilon^{i} \boldsymbol{\tau}^{i}(\hat{\boldsymbol{x}}, \boldsymbol{y})
$$

with

$$
\left\{\begin{array}{l}
\boldsymbol{\tau}^{-1}=\boldsymbol{A} \boldsymbol{\gamma}_{y}\left(\boldsymbol{v}^{0}\right) \\
\boldsymbol{\tau}^{i}=\boldsymbol{A} \boldsymbol{\gamma}_{x}\left(\boldsymbol{v}^{i}\right)+\boldsymbol{A} \boldsymbol{\gamma}_{y}\left(\boldsymbol{v}^{i+1}\right) \text { for } \quad i \geq 0
\end{array}\right.
$$

- in $I$ :

$$
\boldsymbol{\sigma}^{\varepsilon}(\boldsymbol{x})=\sum_{i=p-1}^{\infty} \varepsilon^{i} \boldsymbol{\tau}^{i}(\hat{\boldsymbol{x}}, \boldsymbol{y})
$$

with

$$
\left\{\begin{array}{l}
\boldsymbol{\tau}^{p-1}=\boldsymbol{A}^{I} \boldsymbol{\gamma}_{y}\left(\boldsymbol{v}^{0}\right) \\
\boldsymbol{\tau}^{i}=\boldsymbol{A}^{I} \boldsymbol{\gamma}_{x}\left(\boldsymbol{v}^{i-p}\right)+\boldsymbol{A}^{I} \boldsymbol{\gamma}_{y}\left(\boldsymbol{v}^{i-p+1}\right) \text { for } i \geq p
\end{array}\right.
$$

The fields $\boldsymbol{\tau}^{i}$ are $\hat{Y}$-periodic, with respect to $\hat{\boldsymbol{y}}$ and $\hat{\boldsymbol{x}} \in \omega$ has to be considered as a parameter. 


\subsection{Deduction of the boundary value problems in the outer and in the inner domain}

Using the equations of the problem (2.2) we find the boundary value problems that $\boldsymbol{u}^{i}$ and $\boldsymbol{v}^{i}$ must satisfy. We also prove that $\boldsymbol{v}^{i}$ are completely determined (eventually up to a constant vector) by the boundary value problem set in the unit cell $Y$; on the contrary the boundary value problems for $\boldsymbol{u}^{i}$ is not completely determined in the global domain $\Omega$ since one needs some transmission conditions on $\omega$. These transmission conditions follow from the matching conditions. More precisely, identifying the terms with the same powers of $\varepsilon$ we obtain the following family of equilibrium equations:

Equilibrium equations

\begin{tabular}{|c|c|}
\hline Order & In: $I$ \\
\hline$p-2$ & $\boldsymbol{d i v}_{y} \boldsymbol{\tau}^{p-1}=\mathbf{0}$ \\
\hline$i \geq p-1$ & $\boldsymbol{d i v}_{x} \boldsymbol{\tau}^{i}+\boldsymbol{d i v}_{y} \boldsymbol{\tau}^{i+1}=0$ \\
\hline
\end{tabular}

\begin{tabular}{|c|c|c|}
\hline Order & In: $\Omega \backslash \omega$ & In: $Y \backslash I$ \\
\hline-2 & $\times$ & $\boldsymbol{d i v}_{y} \boldsymbol{\tau}^{-1}=0$ \\
\hline-1 & $\times$ & $\boldsymbol{d i v}_{x} \boldsymbol{\tau}^{-1}+\boldsymbol{d i v}_{y} \boldsymbol{\tau}^{0}=\mathbf{0}$ \\
\hline$i \geq 0$ & $\boldsymbol{d i v}_{x} \boldsymbol{\sigma}^{\boldsymbol{i}}=0$ & $\boldsymbol{d i v}_{x} \boldsymbol{\tau}^{i}+\boldsymbol{d i v}_{y} \boldsymbol{\tau}^{i+1}=\mathbf{0}$ \\
\hline
\end{tabular}

and boundary conditions

Boundary conditions

\begin{tabular}{|c|c|}
\hline Order & Outer \\
\hline$i=0$ & $\begin{array}{c}\boldsymbol{\sigma}^{0} \boldsymbol{n}=\boldsymbol{F} \text { on } \Gamma_{F} \\
\boldsymbol{u}^{0}=\mathbf{0} \text { on } \Gamma_{0}\end{array}$ \\
\hline$i>0$ & $\begin{array}{c}\boldsymbol{\sigma}^{i} \boldsymbol{n}=\mathbf{0} \text { on } \Gamma_{F} \\
\boldsymbol{u}^{i}=\mathbf{0} \text { on } \Gamma_{0}\end{array}$ \\
\hline
\end{tabular}

and for every $\hat{\boldsymbol{x}} \in \omega$ and every $y_{1} \in \mathbb{R}$ the periodicity conditions with respect to $\hat{\boldsymbol{y}} \in \hat{Y}$. These periodicity conditions can be written in the following way:

\begin{tabular}{|c|c|}
\hline$i \geq-1$ & $\boldsymbol{\tau}^{i}\left(\hat{\boldsymbol{x}}, y_{1}, \frac{\alpha}{2}, y_{3}\right) \boldsymbol{e}_{2}=\boldsymbol{\tau}^{i}\left(\hat{\boldsymbol{x}}, y_{1}, \frac{-\alpha}{2}, y_{3}\right) \boldsymbol{e}_{2}$ \\
& $\boldsymbol{\tau}^{i}\left(\hat{\boldsymbol{x}}, y_{1}, y_{2}, \frac{\alpha}{2}\right) \boldsymbol{e}_{3}=\boldsymbol{\tau}^{i}\left(\hat{\boldsymbol{x}}, y_{1}, y_{2}, \frac{-\alpha}{2}\right) \boldsymbol{e}_{3}$ \\
\hline$i \geq 0$ & $\boldsymbol{v}^{i}\left(\hat{\boldsymbol{x}}, y_{1}, \frac{\alpha}{2}, y_{3}\right)=\boldsymbol{v}^{i}\left(\hat{\boldsymbol{x}}, y_{1}, \frac{-\alpha}{2}, y_{3}\right)$ \\
& $\boldsymbol{v}^{i}\left(\hat{\boldsymbol{x}}, y_{1}, y_{2}, \frac{\alpha}{2}\right)=\boldsymbol{v}^{i}\left(\hat{\boldsymbol{x}}, y_{1}, y_{2}, \frac{-\alpha}{2}\right)$ \\
\hline
\end{tabular}

The previous conditions have to be supplemented for every $\hat{\boldsymbol{x}} \in \omega$ with the inner transmission conditions on $\partial I$. In order to avoid ambiguities when useful we use the subscript + , resp. - , to indicate the restriction from outside, resp. inside, $I$. 


\begin{tabular}{|l|l|c|}
\hline & Order & On $\partial I$ \\
\hline if $p=0$ & $i \geq-1$ & {$\left[\boldsymbol{\tau}^{i} \boldsymbol{n}\right]_{\partial I}=\left[\boldsymbol{v}^{i+1}\right]_{\partial I}=\mathbf{0}$} \\
\hline if $p>0$ & $i=-1, \ldots, p-2$ & $\boldsymbol{\tau}_{+}^{i} \boldsymbol{n}=\mathbf{0}$ \\
& $i \geq p-1$ & {$\left[\boldsymbol{\tau}^{i} \boldsymbol{n}\right]_{\partial I}=\left[\boldsymbol{v}^{i-p+1}\right]_{\partial I}=\mathbf{0}$} \\
\hline
\end{tabular}

\subsection{Introduction of the matching conditions}

Since the inner and the outer expansions represent the same functions they have to coincide in the intermediate region $\Omega^{m, \varepsilon}$, cf figure 6 . In order to achieve this requirement one makes at first a Taylor development with respect to $x_{1}$ of each term of the outer expansion near $\omega$ (i.e. for $x_{1}=0$ ). In this way one obtains for instance for every $\mathbf{u}^{i}$ and for $\frac{\varepsilon}{2}<x_{1}<\frac{\eta(\varepsilon)}{2}$ :

$$
\mathbf{u}^{i}(\mathbf{x})=\mathbf{u}^{i}(0+, \hat{\boldsymbol{x}})+x_{1} \frac{\partial \mathbf{u}^{i}}{\partial x_{1}}(0+, \hat{\boldsymbol{x}})+\ldots
$$

Hence since $x_{1}=\varepsilon y_{1}$ one obtains rearranging the terms:

$$
\mathbf{u}^{\varepsilon}\left(x_{1}, \hat{\boldsymbol{x}}\right)=\mathbf{u}^{0}(0+, \hat{\boldsymbol{x}})+\varepsilon\left(\mathbf{u}^{1}(0+, \hat{\boldsymbol{x}})+y_{1} \frac{\partial \mathbf{u}^{0}}{\partial x_{1}}(0+, \hat{\boldsymbol{x}})\right)+\ldots
$$

In $\Omega^{m, \varepsilon}$ one has also :

$$
\mathbf{u}^{\varepsilon}\left(x_{1}, \hat{\boldsymbol{x}}\right)=\mathbf{v}^{0}\left(\hat{\boldsymbol{x}}, y_{1}, \hat{\boldsymbol{y}}\right)+\varepsilon \mathbf{v}^{1}\left(\hat{\boldsymbol{x}}, y_{1}, \hat{\boldsymbol{y}}\right)+\ldots \ldots
$$

Remarking that $\Omega^{m, \varepsilon}=\left\{\boldsymbol{x} \in \Omega ; \frac{1}{2}<\left|y_{1}\right|=\frac{\left|x_{1}\right|}{\varepsilon}<\frac{\eta(\varepsilon)}{2 \varepsilon}\right\}$ one can identify each power of $\varepsilon$ of the outer expansion with the corresponding power of the inner expansion. As the image of the intermediate region in the coordinates $\left(x_{1}, x_{2}, x_{3}\right)$ of the outer domain tends for $\varepsilon \rightarrow 0$ to $\left\{\boldsymbol{x}=\left(x_{1}, x_{2}, x_{3}\right) ; x_{1}=0\right\}(=\omega)$, and in the coordinates of the inner domain to the unbounded domain $\left\{\boldsymbol{y}=\left(y_{1}, y_{2}, y_{3}\right) ;\left|y_{1}\right|>1\right\}$, the matching conditions will be for each power of $\varepsilon$ and for every $\hat{\boldsymbol{x}} \in \omega$ and every $\hat{\boldsymbol{y}} \in \hat{Y}$ :

$$
\begin{aligned}
& \text { limit for } x_{1} \text { going to } 0 \pm \text { of the outer expansion }= \\
& \text { limit for } y_{1} \text { going to } \pm \infty \text { of the inner expansion }
\end{aligned}
$$

Following Ref. 25 (or Ref. 23) these conditions correspond to the assumption:

$$
\text { inner limit of the outer limit }=\text { outer limit of the inner limit. }
$$

In our situation we only use the following matching conditions to be satisfied for every $\hat{\boldsymbol{x}} \in \omega$ and every $\hat{\boldsymbol{y}} \in \hat{Y}$ :

$$
\begin{gathered}
\lim _{y_{1} \rightarrow \pm \infty} \boldsymbol{\tau}^{-1}\left(\hat{\boldsymbol{x}}, y_{1}, \hat{\boldsymbol{y}}\right) \boldsymbol{e}_{1}=\mathbf{0} \\
\lim _{y_{1} \rightarrow \pm \infty} \boldsymbol{v}^{0}\left(\hat{\boldsymbol{x}}, y_{1}, \hat{\boldsymbol{y}}\right)=\boldsymbol{u}^{0}(0 \pm, \hat{\boldsymbol{x}})
\end{gathered}
$$




$$
\begin{gathered}
\lim _{y_{1} \rightarrow \pm \infty} \boldsymbol{\tau}^{0}\left(\hat{\boldsymbol{x}}, y_{1}, \hat{\boldsymbol{y}}\right) \boldsymbol{e}_{1}=\boldsymbol{\sigma}^{0}(0 \pm, \hat{\boldsymbol{x}}) \boldsymbol{e}_{1} \\
\lim _{y_{1} \rightarrow \pm \infty}\left(\boldsymbol{v}^{1}\left(\hat{\boldsymbol{x}}, y_{1}, \hat{\boldsymbol{y}}\right)-\boldsymbol{u}^{1}(0 \pm, \hat{\boldsymbol{x}})-y_{1} \frac{\partial \boldsymbol{u}^{0}}{\partial x_{1}}(0 \pm, \hat{\boldsymbol{x}})\right)=\mathbf{0} \\
\lim _{y_{1} \rightarrow \pm \infty}\left(\boldsymbol{\tau}^{1}\left(\hat{\boldsymbol{x}}, y_{1}, \hat{\boldsymbol{y}}\right) \boldsymbol{e}_{1}-\boldsymbol{\sigma}^{1}(0 \pm, \hat{\boldsymbol{x}}) \boldsymbol{e}_{1}-y_{1} \frac{\partial \boldsymbol{\sigma}^{0}}{\partial x_{1}}(0 \pm, \hat{\boldsymbol{x}}) \boldsymbol{e}_{1}\right)=\mathbf{0}
\end{gathered}
$$

\subsection{Definition of the outer and inner approximations}

Once determined the different terms $\mathbf{u}^{i}$ and $\mathbf{v}^{i}$ one can define for every $m \geq 0$ an outer and an inner approximation of order $m$ :

$$
\begin{aligned}
& \mathbf{u}_{m}^{\text {out }}=\sum_{i=0}^{i=m} \varepsilon^{i} \mathbf{u}^{i} \\
& \mathbf{u}_{m}^{i n n}=\sum_{i=0}^{i=m} \varepsilon^{i} \mathbf{v}^{i}
\end{aligned}
$$

The quality of these approximations increases with $m$. In the following we compute only the first two terms $\mathbf{u}^{i}$ and $\mathbf{v}^{i}$ for $i=0,1$ and hence the corresponding outer and inner approximations of order $m=0,1$. In Section 5 the numerical experiments will prove the gain of precision that is obtained increasing the value of $m$ from $m=0$ to $m=1$.

\section{The two first orders outer approximations}

\subsection{Zero order outer approximation}

Theorem 4.1. The zero order outer approximation $\left(\boldsymbol{u}^{0}, \boldsymbol{\sigma}^{0}\right)$ is the solution of the following transmission linear problem :

$$
\begin{cases}\boldsymbol{d i v} \boldsymbol{\sigma}^{0}=\mathbf{0} & \text { in } \Omega \\ \boldsymbol{\sigma}^{0}=\boldsymbol{A} \boldsymbol{\gamma}\left(\boldsymbol{u}^{0}\right) & \text { in } \Omega \\ \boldsymbol{\sigma}^{0} \boldsymbol{n}=\boldsymbol{F} & \text { on } \Gamma_{F} \\ \boldsymbol{u}^{0}=\mathbf{0} & \text { on } \Gamma_{0}\end{cases}
$$

Remark 4.1. Let us state explicitly that in the zero order outer approximation the heterogeneities have completely disappeared and that the problem has a unique solution $\boldsymbol{u}^{0} \in \boldsymbol{H}^{1}(\Omega)$. Moreover the solution $\boldsymbol{u}^{0}$ is regular when the data are regular and in particular its trace on $\omega$ will be also regular. 


\subsubsection{Determination of $\boldsymbol{v}^{0}$}

Lemma 4.1. For $p \geq 0$ one has $\boldsymbol{v}^{0}\left(\hat{\boldsymbol{x}}, y_{1}, \hat{\boldsymbol{y}}\right)=\boldsymbol{v}^{0}(\hat{\boldsymbol{x}})$ and hence

$$
\lim _{y_{1} \rightarrow-\infty} \boldsymbol{v}^{0}\left(\hat{\boldsymbol{x}}, y_{1}, \hat{\boldsymbol{y}}\right)=\lim _{y_{1} \rightarrow+\infty} \boldsymbol{v}^{0}\left(\hat{\boldsymbol{x}}, y_{1}, \hat{\boldsymbol{y}}\right)=\boldsymbol{v}^{0}(\hat{\boldsymbol{x}})
$$

Proof. In the case $p=0, \boldsymbol{v}^{0}$ and $\boldsymbol{\tau}^{-1}$ are solutions for every $\hat{\boldsymbol{x}} \in \omega$ of the following problem :

$$
\begin{cases}\boldsymbol{d i v}_{y} \boldsymbol{\tau}^{-1}=\mathbf{0} & \text { in } Y \\ \boldsymbol{\tau}^{-1}=\tilde{\boldsymbol{A}} \boldsymbol{\gamma}_{y}\left(\boldsymbol{v}^{\mathbf{0}}\right) & \text { in } Y \\ \boldsymbol{\tau}^{-1}\left(\hat{\boldsymbol{x}}, y_{1}, \frac{\alpha}{2}, y_{3}\right) \boldsymbol{e}_{2}=\boldsymbol{\tau}^{-1}\left(\hat{\boldsymbol{x}}, y_{1}, \frac{-\alpha}{2}, y_{3}\right) \boldsymbol{e}_{2} & \text { on } \mathbb{R} \times \partial \hat{Y} \\ \boldsymbol{\tau}^{-1}\left(\hat{\boldsymbol{x}}, y_{1}, y_{2}, \frac{\alpha}{2}\right) \boldsymbol{e}_{3}=\boldsymbol{\tau}^{-1}\left(\hat{\boldsymbol{x}}, y_{1}, y_{2}, \frac{-\alpha}{2}\right) \boldsymbol{e}_{3} & \text { on } \mathbb{R} \times \partial \hat{Y} \\ \boldsymbol{v}^{0}\left(\hat{\boldsymbol{x}}, y_{1}, \frac{\alpha}{2}, y_{3}\right)=\boldsymbol{v}^{0}\left(\hat{\boldsymbol{x}}, y_{1}, \frac{-\alpha}{2}, y_{3}\right) & \text { on } \mathbb{R} \times \partial \hat{Y} \\ \boldsymbol{v}^{0}\left(\hat{\boldsymbol{x}}, y_{1}, y_{2}, \frac{\alpha}{2}\right)=\boldsymbol{v}^{0}\left(\hat{\boldsymbol{x}}, y_{1}, y_{2}, \frac{-\alpha}{2}\right) & \text { on } \mathbb{R} \times \partial \hat{Y} \\ \lim _{y_{1} \rightarrow \pm \infty} \boldsymbol{\tau}^{-1}\left(\hat{\boldsymbol{x}}, y_{1}, \hat{\boldsymbol{y}}\right) \boldsymbol{e}_{1}=\mathbf{0} & \text { for } \hat{\boldsymbol{y}} \in \hat{Y}\end{cases}
$$

with

$$
\tilde{\boldsymbol{A}}= \begin{cases}\boldsymbol{A} & \text { in } Y \backslash I \\ \boldsymbol{A}^{I} & \text { in } I\end{cases}
$$

It follows that $\boldsymbol{\tau}^{-1}=\tilde{\boldsymbol{A}} \boldsymbol{\gamma}_{y}\left(\boldsymbol{v}^{0}\right)=\mathbf{0}$ and hence $\boldsymbol{v}^{0}\left(\hat{\boldsymbol{x}}, y_{1}, \hat{\boldsymbol{y}}\right)=\boldsymbol{v}^{0}(\hat{\boldsymbol{x}})$ and (4.2).

When $p>0$, the proof is essentially the same. One has only to remark that $\boldsymbol{\tau}^{-1}=\mathbf{0}$ in $I$ and hence $\boldsymbol{v}^{0}$ and $\boldsymbol{\tau}^{-1}$ are solutions in $Y \backslash I$ of a problem analogous to (4.3). Therefore in $Y \backslash I$ one has $\boldsymbol{\tau}^{-1}=\boldsymbol{A} \gamma_{y}\left(\boldsymbol{v}^{0}\right)=\mathbf{0}$ and so $\boldsymbol{v}^{0}\left(\hat{\boldsymbol{x}}, y_{1}, \hat{\boldsymbol{y}}\right)=\boldsymbol{v}^{0}(\hat{\boldsymbol{x}})$. As a consequence (4.2) holds true also in this case.

\subsubsection{Proof of Theorem 4.1}

Thanks to 3.5 it is enough to prove only that on $\omega$ one has $\left[\boldsymbol{u}^{0}\right]_{\omega}=\mathbf{0}$ and $\left[\boldsymbol{\sigma}^{0} \boldsymbol{e}_{1}\right]_{\omega}=$ 0. These follow from the matching conditions (3.15) and (3.16). Let us remark at first that (4.2) and the matching condition (3.15) imply that $\left[\boldsymbol{u}^{0}\right]_{\omega}=\mathbf{0}$. In order to prove the continuity of $\boldsymbol{\sigma}^{0} \boldsymbol{e}_{1}$ through $\omega$, one remarks that for $p \geq 0$ one has in all $\omega \times Y$ :

$$
\boldsymbol{d i v} \boldsymbol{v}_{y} \boldsymbol{\tau}^{0}=\mathbf{0}
$$

and then that the matching condition (3.16) can also be written as follows:

$$
|\hat{Y}|\left[\boldsymbol{\sigma}^{0} \boldsymbol{e}_{1}\right]_{\omega}=\lim _{L \rightarrow+\infty}\left(\int_{\hat{Y}}\left(\boldsymbol{\tau}^{0}(\hat{\boldsymbol{x}}, L, \hat{\boldsymbol{y}}) \boldsymbol{e}_{1}-\boldsymbol{\tau}^{0}(\hat{\boldsymbol{x}},-L, \hat{\boldsymbol{y}}) \boldsymbol{e}_{1}\right) d \hat{\boldsymbol{y}}\right)
$$

Therefore integrating the equation (4.5) on $\left.Y_{L}:=\right]-L, L[\times \hat{Y}$, from the Green formula and the periodicity conditions on $\tau^{0}$ one deduces for every $L>0$ :

$$
\int_{\hat{Y}}\left(\boldsymbol{\tau}^{0}(\hat{\boldsymbol{x}}, L, \hat{\boldsymbol{y}}) \boldsymbol{e}_{1}-\boldsymbol{\tau}^{0}(\hat{\boldsymbol{x}},-L, \hat{\boldsymbol{y}}) \boldsymbol{e}_{1}\right) d \hat{\boldsymbol{y}}=\mathbf{0}
$$


and hence $\left[\boldsymbol{\sigma}^{0} \boldsymbol{e}_{1}\right]_{\omega}=\mathbf{0}$.

Corollary 4.1. One has $\boldsymbol{v}^{0}(\hat{\boldsymbol{x}})=\boldsymbol{u}^{0}(0, \hat{\boldsymbol{x}})$.

The result follows immediately from (4.2) and the matching condition (3.15).

\subsection{First order outer approximation}

The outer equilibrium and constitutive equations and the boundary conditions imply that $\boldsymbol{u}^{1}$ and $\boldsymbol{\sigma}^{1}$ are solutions of the following set of equations:

$$
\begin{cases}\boldsymbol{d i v} \boldsymbol{\sigma}^{1}=\mathbf{0} & \text { in } \Omega \backslash \omega \\ \boldsymbol{\sigma}^{1}=\boldsymbol{A} \boldsymbol{\gamma}\left(\boldsymbol{u}^{1}\right) & \text { in } \Omega \backslash \omega \\ \boldsymbol{\sigma}^{1} \boldsymbol{n}=\mathbf{0} & \text { on } \Gamma_{F} \\ \boldsymbol{u}^{1}=\mathbf{0} & \text { on } \Gamma_{0}\end{cases}
$$

In order to obtain a well-posed boundary value problem we need to find the transmission conditions on $\omega$. The matching conditions (3.17), (3.18) and the Proposition 4.1 imply that these transmission conditions are the following:

$$
\begin{aligned}
& {\left[\boldsymbol{u}^{1}\right]_{\omega}=} \\
& \lim _{y_{1} \rightarrow+\infty}\left(\frac{1}{|\hat{Y}|} \int_{\hat{Y}}\left(\boldsymbol{v}^{1}\left(\hat{\boldsymbol{x}}, y_{1}, \hat{\boldsymbol{y}}\right)-\boldsymbol{v}^{1}\left(\hat{\boldsymbol{x}},-y_{1}, \hat{\boldsymbol{y}}\right)\right) d \hat{\boldsymbol{y}}-2 y_{1} \frac{\partial \boldsymbol{u}^{0}}{\partial x_{1}}(0, \hat{\boldsymbol{x}})\right) \\
& {\left[\boldsymbol{\sigma}^{1} \boldsymbol{e}_{1}\right]_{\omega}=} \\
& \lim _{y_{1} \rightarrow+\infty}\left(\frac{1}{|\hat{Y}|} \int_{\hat{Y}}\left(\boldsymbol{\tau}^{1}\left(\hat{\boldsymbol{x}}, y_{1}, \hat{\boldsymbol{y}}\right) \boldsymbol{e}_{1}-\boldsymbol{\tau}^{1}\left(\hat{\boldsymbol{x}},-y_{1}, \hat{\boldsymbol{y}}\right) \boldsymbol{e}_{1}\right) d \hat{\boldsymbol{y}}-2 y_{1} \frac{\partial \boldsymbol{\sigma}^{0}}{\partial x_{1}}(0, \hat{\boldsymbol{x}}) \boldsymbol{e}_{1}\right)
\end{aligned}
$$

From the Green formula and the periodicity conditions one finds

$$
\int_{\hat{Y}}\left(\boldsymbol{\tau}^{1}(\hat{\boldsymbol{x}}, L, \hat{\boldsymbol{y}}) \boldsymbol{e}_{1}-\boldsymbol{\tau}^{1}(\hat{\boldsymbol{x}},-L, \hat{\boldsymbol{y}}) \boldsymbol{e}_{1}\right) d \hat{\boldsymbol{y}}=\int_{Y_{L}} \boldsymbol{d i v}_{y} \boldsymbol{\tau}^{1}(\hat{\boldsymbol{x}}, \boldsymbol{y}) d \boldsymbol{y}
$$

and hence the inner equilibrium equation of order 0 implies that it is enough to completely determine $\tau^{0}$.

\subsubsection{Determination of $\boldsymbol{v}^{1}$ and of $\boldsymbol{\tau}^{0}$}

The transmission condition (4.9) being a limit condition for $y_{1} \rightarrow+\infty$ for every $\hat{\boldsymbol{x}} \in \omega$ we shall decompose $\boldsymbol{v}^{1}$ as follows:

$$
\boldsymbol{v}^{1}(\hat{\boldsymbol{x}}, \boldsymbol{y})=f\left(y_{1} ; a, b\right) \frac{\partial \boldsymbol{u}^{0}}{\partial x_{1}}(0, \hat{\boldsymbol{x}})+\boldsymbol{v}^{\star}(\hat{\boldsymbol{x}}, \boldsymbol{y})
$$

where $f\left(y_{1} ; a, b\right)$ is a smooth odd function (e.g. of class $\left.\mathcal{C}^{2}(\mathbb{R})\right)$ with:

$$
f\left(y_{1} ; a, b\right)= \begin{cases}0 & \text { if } 0<\left|y_{1}\right| \leq a \\ y_{1} & \text { if }\left|y_{1}\right| \geq b\end{cases}
$$


and $d<2 a<2 b$ (let us also recall that $I \cap\left\{y_{1}=0\right\}$ is strictly contained in $\hat{Y}$, cf. figure 7$)$. In the sequel for simplicity we only write $f\left(y_{1}\right)$.

Proposition 4.1. (i) When $p=0$, let $\tilde{\boldsymbol{A}}$ be defined by $\tilde{\boldsymbol{A}}=\boldsymbol{A}$ in $Y \backslash I$ and by $\tilde{\boldsymbol{A}}=\boldsymbol{A}^{I}$ in $I$; then the displacement field $\boldsymbol{v}^{\star}\left(\hat{\boldsymbol{x}}, y_{1}, \hat{\boldsymbol{y}}\right)$ is, for every $\hat{\boldsymbol{x}} \in \omega$, the unique, up to a translation with respect to $\boldsymbol{y}$, solution of the following problem:

$$
\begin{cases}\boldsymbol{d i v}_{y} \boldsymbol{\tau}^{\star}=-f^{\prime \prime}\left(y_{1}\right) \tilde{\boldsymbol{A}}\left(\frac{\partial \boldsymbol{u}^{0}}{\partial x_{1}}(0, \hat{\boldsymbol{x}}) \otimes_{S} \boldsymbol{e}_{1}\right) \boldsymbol{e}_{1} & \text { in } Y \\ \boldsymbol{\tau}^{\star}=\tilde{\boldsymbol{A}} \boldsymbol{\gamma}_{y}\left(\boldsymbol{v}^{\star}\right) & \text { in } Y \\ \boldsymbol{\tau}^{\star}\left(\hat{\boldsymbol{x}}, y_{1}, \frac{\alpha}{2}, y_{3}\right) \boldsymbol{e}_{2}=\boldsymbol{\tau}^{\star}\left(\hat{\boldsymbol{x}}, y_{1}, \frac{-\alpha}{2}, y_{3}\right) \boldsymbol{e}_{2} & \text { on } \mathbb{R} \times \partial \hat{Y} \\ \boldsymbol{\tau}^{\star}\left(\hat{\boldsymbol{x}}, y_{1}, y_{2}, \frac{\alpha}{2}\right) \boldsymbol{e}_{3}=\boldsymbol{\tau}^{\star}\left(\hat{\boldsymbol{x}}, y_{1}, y_{2}, \frac{-\alpha}{2}\right) \boldsymbol{e}_{3} & \text { on } \mathbb{R} \times \partial \hat{Y} \\ \boldsymbol{v}^{\star}\left(\hat{\boldsymbol{x}}, y_{1}, \frac{\alpha}{2}, y_{3}\right)=\boldsymbol{v}^{\star}\left(\hat{\boldsymbol{x}}, y_{1}, \frac{-\alpha}{2}, y_{3}\right) & \text { on } \mathbb{R} \times \partial \hat{Y} \\ \boldsymbol{v}^{\star}\left(\hat{\boldsymbol{x}}, y_{1}, y_{2}, \frac{\alpha}{2}\right)=\boldsymbol{v}^{\star}\left(\hat{\boldsymbol{x}}, y_{1}, y_{2}, \frac{-\alpha}{2}\right) & \text { on } \mathbb{R} \times \partial \hat{Y} \\ \lim _{y_{1} \rightarrow \pm \infty} \boldsymbol{\tau}^{\star}\left(\hat{\boldsymbol{x}}, y_{1}, \hat{\boldsymbol{y}}\right) \boldsymbol{e}_{1}=\mathbf{0} & \text { for } \hat{\boldsymbol{y}} \in \hat{Y} \\ {\left[\boldsymbol{v}^{\star}\right]_{\partial I}=\mathbf{0}} & \\ {\left[\boldsymbol{\tau}^{\star} \boldsymbol{n}\right]_{\partial I}=\left(\left(\boldsymbol{A}^{I}-\boldsymbol{A}\right) \boldsymbol{\gamma}_{\boldsymbol{x}}\left(\boldsymbol{u}^{0}(0, \hat{\boldsymbol{x}})\right)\right) \boldsymbol{n}} & \end{cases}
$$

(ii) For every $\hat{\boldsymbol{x}} \in \omega$ the displacement field $\boldsymbol{v}^{\star}\left(\hat{\boldsymbol{x}}, y_{1}, \hat{\boldsymbol{y}}\right)$ does not depend when $p>0$ on $p$ and is for every $\hat{\boldsymbol{x}} \in \omega$, the unique, up to a translation with respect to $\boldsymbol{y}$, solution of the following problem:

$$
\begin{cases}\boldsymbol{d i v}_{y} \boldsymbol{\tau}^{\star}=-f^{\prime \prime}\left(y_{1}\right) \boldsymbol{A}\left(\frac{\partial \boldsymbol{u}^{0}}{\partial x_{1}}(0, \hat{\boldsymbol{x}}) \otimes_{S} \boldsymbol{e}_{1}\right) \boldsymbol{e}_{1} & \text { in } Y \backslash I \\ \boldsymbol{\tau}^{\star}=\boldsymbol{A} \boldsymbol{\gamma}_{y}\left(\boldsymbol{v}^{\star}\right) & \text { in } Y \backslash I \\ \boldsymbol{\tau}^{\star}\left(\hat{\boldsymbol{x}}, y_{1}, \frac{\alpha}{2}, y_{3}\right) \boldsymbol{e}_{2}=\boldsymbol{\tau}^{\star}\left(\hat{\boldsymbol{x}}, y_{1}, \frac{-\alpha}{2}, y_{3}\right) \boldsymbol{e}_{2} & \text { on } \mathbb{R} \times \partial \hat{Y} \\ \boldsymbol{\tau}^{\star}\left(\hat{\boldsymbol{x}}, y_{1}, y_{2}, \frac{\alpha}{2}\right) \boldsymbol{e}_{3}=\boldsymbol{\tau}^{\star}\left(\hat{\boldsymbol{x}}, y_{1}, y_{2}, \frac{-\alpha}{2}\right) \boldsymbol{e}_{3} & \text { on } \mathbb{R} \times \partial \hat{Y} \\ \boldsymbol{v}^{\star}\left(\hat{\boldsymbol{x}}, y_{1}, \frac{\alpha}{2}, y_{3}\right)=\boldsymbol{v}^{\star}\left(\hat{\boldsymbol{x}}, y_{1}, \frac{-\alpha}{2}, y_{3}\right) & \text { on } \mathbb{R} \times \partial \hat{Y} \\ \boldsymbol{v}^{\star}\left(\hat{\boldsymbol{x}}, y_{1}, y_{2}, \frac{\alpha}{2}\right)=\boldsymbol{v}^{\star}\left(\hat{\boldsymbol{x}}, y_{1}, y_{2}, \frac{-\alpha}{2}\right) & \text { on } \mathbb{R} \times \partial \hat{Y} \\ \lim _{y_{1} \rightarrow \pm \infty} \boldsymbol{\tau}^{\star}\left(\hat{\boldsymbol{x}}, y_{1}, \hat{\boldsymbol{y}}\right) \boldsymbol{e}_{1}=\mathbf{0} & \text { for } \hat{\boldsymbol{y}} \in \hat{Y} \\ \boldsymbol{\tau}_{+}^{\star} \boldsymbol{n}=-\boldsymbol{A}\left(\frac{\partial u^{0}}{\partial x_{2}}(0, \hat{\boldsymbol{x}}) \otimes_{S} \boldsymbol{e}_{2}+\frac{\partial u^{0}}{\partial x_{3}}(0, \hat{\boldsymbol{x}}) \otimes_{S} \boldsymbol{e}_{3}\right) \boldsymbol{n} & \text { on } \partial I\end{cases}
$$

(iii) For every $\hat{\boldsymbol{x}} \in \omega$ when $p>0$ the displacement field $\boldsymbol{v}^{1}\left(\hat{\boldsymbol{x}}, y_{1}, \hat{\boldsymbol{y}}\right)$ does not depend on $p$ and is the unique solution of the Dirichlet boundary value problem:

$$
\begin{cases}\boldsymbol{d i v}_{y} \boldsymbol{A}^{I} \boldsymbol{\gamma}_{y}\left(\boldsymbol{v}^{1}\right)=0 & \text { in } I \\ \boldsymbol{v}^{1}=\boldsymbol{v}^{\star} & \text { on } \partial I\end{cases}
$$

(iv) For all $p \geq 0$ the following asymptotic behaviour holds true:

$$
\lim _{y_{1} \rightarrow \pm \infty} \int_{\hat{Y}} \boldsymbol{v}^{\star}\left(\hat{\boldsymbol{x}}, y_{1}, \hat{\boldsymbol{y}}\right) d \hat{\boldsymbol{y}}=\boldsymbol{v}^{\star \pm}(\hat{\boldsymbol{x}})
$$


Proof. (i), (ii) The first equations and the limit behaviour of $\boldsymbol{\tau}^{\star}$ in the case $p>0$ (resp. $p=0$ ) follow immediately from the equilibrium of order $p-1$ (resp. -1 ), the constitutive equations of order $i=p$ (resp. $i=0$ ), the periodicity conditions, the choice of $f\left(y_{1}\right)$, the property $\boldsymbol{v}^{0}\left(\hat{\boldsymbol{x}}, y_{1}, \hat{\boldsymbol{y}}\right)=\boldsymbol{v}^{0}(\hat{\boldsymbol{x}})=\boldsymbol{u}^{0}(0, \hat{\boldsymbol{x}})$ and the transmission conditions of order $p-1$ (resp. -1 ) on $\partial I$.

(iii) The boundary value problem (4.16) on $\partial I$ follows from the equilibrium equations of order $p-2$ and $p-1$, the constitutive equations (3.12) of order $i=p-1$ and $i=p$, the Corollary 4.1, the transmission condition of order $p$ and the decomposition (4.12). Let us remark that the solution of (4.16) is uniquely determined; hence when a translation with respect to $\boldsymbol{y}$ is added to $\boldsymbol{v}^{\star}$ in $Y \backslash I$ then the same translation will be added to $\boldsymbol{v}^{1}$ in $I$.

(iv) In order to prove (4.17) let us only remark that the assumptions on $f\left(y_{1}\right)$ imply that $\boldsymbol{d i} \boldsymbol{v}_{y} \boldsymbol{\tau}^{\star}=\mathbf{0}$ for $\left|y_{1}\right|>b$ and hence for a result on the analyticity of the solutions of the linear elasticity system (see e.g. Gurtin ${ }^{14}$, Sect. 27 in the isotropic case ) one has that $\boldsymbol{v}^{\star}\left(\hat{\boldsymbol{x}}, y_{1}, \hat{\boldsymbol{y}}\right)$ is an analytic function of $\boldsymbol{y}$ in the strip $\left|y_{1}\right|>b$. The periodicity conditions and the limit behaviour of $\boldsymbol{\tau}^{\star}\left(\hat{\boldsymbol{x}}, y_{1}, \hat{\boldsymbol{y}}\right) \boldsymbol{e}_{1}$ imply the result.

Remark 4.2. The problem (4.15) coincides with the problem obtained in the case of the holes in Ref. 9. Hence in order to simplify the statements in the following proposition 4.2 we only consider the case $p=0$. The corresponding statements for $p>0$ can be deduced with obvious modifications; let us only remark that from the decomposition of $\boldsymbol{v}^{\star}$ in $\omega \times Y \backslash I$ it follows easily the decomposition of $\boldsymbol{v}^{1}$ in $\omega \times I$.

The linearity of problem (4.14) implies a decomposition in nine elementary universal problems.

Proposition 4.2. For every $\hat{\boldsymbol{x}} \in \omega$, the solution $\boldsymbol{v}^{\star}$ of the problem (4.14) can be decomposed as follows:

$$
\boldsymbol{v}^{\star}(\hat{\boldsymbol{x}}, \boldsymbol{y})=\frac{\partial u_{l}^{0}}{\partial x_{k}}(0, \hat{\boldsymbol{x}}) \boldsymbol{V}^{l k}(\boldsymbol{y})+\check{\boldsymbol{v}}(\hat{\boldsymbol{x}})
$$

Here $\check{\boldsymbol{v}}$ is a constant (with respect to $\boldsymbol{y}$ ) displacement field and $\left(\boldsymbol{V}^{l k}(\boldsymbol{y}), \boldsymbol{T}^{l k}(\boldsymbol{y})\right)$ are the unique solutions satisfying the asymptotic condition $\boldsymbol{V}^{i j+}+\boldsymbol{V}^{i j-}=\mathbf{0}$, whith $\boldsymbol{V}^{i j \pm}:=\lim _{y_{1} \rightarrow \pm \infty} \int_{\hat{Y}} \boldsymbol{V}^{i j}\left(y_{1}, \hat{\boldsymbol{y}}\right) d \hat{\boldsymbol{y}}$, of the following elementary problems : 
i) for $(l, 1), l=1,2,3$ :

$$
\begin{cases}\boldsymbol{d i v}_{y} \boldsymbol{T}^{l 1}=-f^{\prime \prime}\left(y_{1}\right) \tilde{A}_{i 1 l 1} \boldsymbol{e}_{i} & \text { in } Y \\ \boldsymbol{T}^{l 1}=\tilde{\boldsymbol{A}} \boldsymbol{\gamma}_{y}\left(\boldsymbol{V}^{l 1}\right) & \text { in } Y \\ \boldsymbol{T}^{l 1}\left(\hat{\boldsymbol{x}}, y_{1}, \frac{\alpha}{2}, y_{3}\right) \boldsymbol{e}_{2}=\boldsymbol{T}^{l 1}\left(\hat{\boldsymbol{x}}, y_{1}, \frac{-\alpha}{2}, y_{3}\right) \boldsymbol{e}_{2} & \text { on } \mathbb{R} \times \partial \hat{Y} \\ \boldsymbol{T}^{l 1}\left(\hat{\boldsymbol{x}}, y_{1}, y_{2}, \frac{\alpha}{2}\right) \boldsymbol{e}_{3}=\boldsymbol{T}^{l 1}\left(\hat{\boldsymbol{x}}, y_{1}, y_{2}, \frac{-\alpha}{2}\right) \boldsymbol{e}_{3} & \text { on } \mathbb{R} \times \partial \hat{Y} \\ \boldsymbol{V}^{l 1}\left(\hat{\boldsymbol{x}}, y_{1}, \frac{\alpha}{2}, y_{3}\right)=\boldsymbol{V}^{l 1}\left(\hat{\boldsymbol{x}}, y_{1}, \frac{-\alpha}{2}, y_{3}\right) & \text { on } \mathbb{R} \times \partial \hat{Y} \\ \boldsymbol{V}^{l 1}\left(\hat{\boldsymbol{x}}, y_{1}, y_{2}, \frac{\alpha}{2}\right)=\boldsymbol{V}^{l 1}\left(\hat{\boldsymbol{x}}, y_{1}, y_{2}, \frac{-\alpha}{2}\right) & \text { on } \mathbb{R} \times \partial \hat{Y} \\ \lim _{y_{1} \rightarrow \pm \infty} \boldsymbol{T}^{l 1}\left(y_{1}, \hat{\boldsymbol{y}}\right) \boldsymbol{e}_{1}=\mathbf{0} & \text { for } \hat{\boldsymbol{y}} \in \hat{Y} \\ {\left[\boldsymbol{V}^{l 1}\right]_{\partial I}=\left[\boldsymbol{T}^{l 1} \boldsymbol{n}\right]_{\partial I}=\mathbf{0}} & \end{cases}
$$

ii) when $(l, k)=(1,2) ;(2,2) ;(3,2) ;(1,3) ;(2,3) ;(3,3)$,

$$
\begin{cases}\boldsymbol{d i v}_{y} \boldsymbol{T}^{l k}=\mathbf{0} & \text { in } Y \\ \boldsymbol{T}^{l k}=\tilde{\boldsymbol{A}} \boldsymbol{\gamma}_{y}\left(\boldsymbol{V}^{l k}\right) & \text { in } Y \\ \boldsymbol{T}^{l k}\left(\hat{\boldsymbol{x}}, y_{1}, \frac{\alpha}{2}, y_{3}\right) \boldsymbol{e}_{2}=\boldsymbol{T}^{l k}\left(\hat{\boldsymbol{x}}, y_{1}, \frac{-\alpha}{2}, y_{3}\right) \boldsymbol{e}_{2} & \text { on } \mathbb{R} \times \partial \hat{Y} \\ \boldsymbol{T}^{l k}\left(\hat{\boldsymbol{x}}, y_{1}, y_{2}, \frac{\alpha}{2}\right) \boldsymbol{e}_{3}=\boldsymbol{T}^{l k}\left(\hat{\boldsymbol{x}}, y_{1}, y_{2}, \frac{-\alpha}{2}\right) \boldsymbol{e}_{3} & \text { on } \mathbb{R} \times \partial \hat{Y} \\ \boldsymbol{V}^{l k}\left(\hat{\boldsymbol{x}}, y_{1}, \frac{\alpha}{2}, y_{3}\right)=\boldsymbol{V}^{l k}\left(\hat{\boldsymbol{x}}, y_{1}, \frac{-\alpha}{2}, y_{3}\right) & \text { on } \mathbb{R} \times \partial \hat{Y} \\ \boldsymbol{V}^{l k}\left(\hat{\boldsymbol{x}}, y_{1}, y_{2}, \frac{\alpha}{2}\right)=\boldsymbol{V}^{l k}\left(\hat{\boldsymbol{x}}, y_{1}, y_{2}, \frac{-\alpha}{2}\right) & \text { on } \mathbb{R} \times \partial \hat{Y} \\ \lim _{y_{1} \rightarrow \pm \infty} \boldsymbol{T}^{l k}\left(y_{1}, \hat{\boldsymbol{y}}\right) \boldsymbol{e}_{1}=\mathbf{0} & \text { for } \hat{\boldsymbol{y}} \in \hat{Y} \\ {\left[\boldsymbol{V}^{l k}\right]_{\partial I}=\mathbf{0}} & \\ {\left[\boldsymbol{T}^{l k} \boldsymbol{n}\right]_{\partial I}=\left(\left(\boldsymbol{A}^{I}-\boldsymbol{A}\right)\left(\boldsymbol{e}_{l} \otimes_{S} \boldsymbol{e}_{k}\right)\right) \boldsymbol{n}} & \end{cases}
$$




\subsubsection{The boundary value problem of the first order outer approximation}

Theorem 4.2. The first order outer approximation $\left(\boldsymbol{u}^{1}, \boldsymbol{\sigma}^{1}\right)$ is the unique solution of the following boundary value problem:

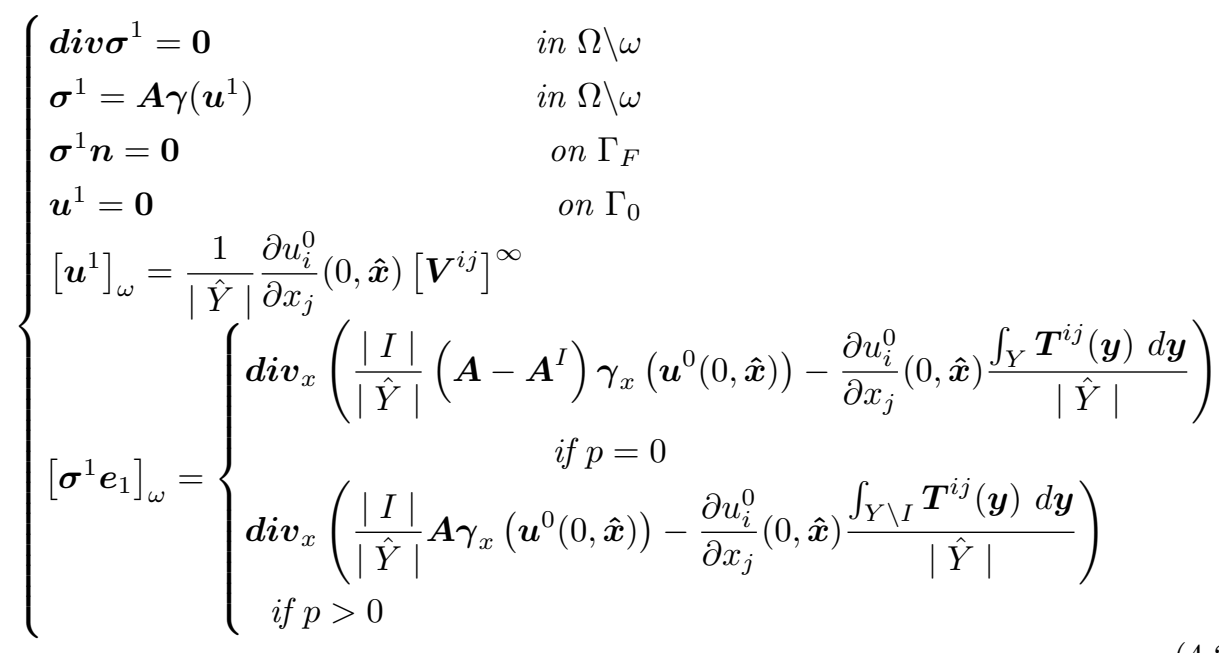

Proof. From (4.9), (4.12) and (4.18) one immediately deduces the first transmission condition on $\omega$ :

$$
\left[\boldsymbol{u}^{1}\right]_{\omega}=\frac{1}{|\hat{Y}|} \frac{\partial u_{i}^{0}}{\partial x_{j}}(0, \hat{\boldsymbol{x}})\left[\boldsymbol{V}^{i j}\right]^{\infty}
$$

where $\left[\boldsymbol{V}^{i j}\right]^{\infty}=\boldsymbol{V}^{i j+}-\boldsymbol{V}^{i j-}$.

In order to find the second transmission condition one remarks that (4.11) and the interior equilibrium equation of order $i=0$ imply:

- for $p=0$

$$
\int_{\hat{Y}}\left(\boldsymbol{\tau}^{1}(\hat{\boldsymbol{x}}, L, \hat{\boldsymbol{y}}) \boldsymbol{e}_{1}-\boldsymbol{\tau}^{1}(\hat{\boldsymbol{x}},-L, \hat{\boldsymbol{y}}) \boldsymbol{e}_{1}\right) d \hat{\boldsymbol{y}}=-\int_{Y_{L}} \boldsymbol{d i \boldsymbol { v } _ { x }} \boldsymbol{\tau}^{0}(\hat{\boldsymbol{x}}, \boldsymbol{y}) d \boldsymbol{y}
$$

since $\boldsymbol{d i v}_{x}\left(\boldsymbol{\sigma}^{0}(0, \hat{\boldsymbol{x}})\right)=\frac{\partial \boldsymbol{\sigma}^{0}}{\partial x_{2}}(0, \hat{\boldsymbol{x}}) \boldsymbol{e}_{2}+\frac{\partial \boldsymbol{\sigma}^{0}}{\partial x_{3}}(0, \hat{\boldsymbol{x}}) \boldsymbol{e}_{3}$, the equation $(4.1)_{1}$ and the proposition 4.2 imply :

$$
\begin{aligned}
& \int_{\hat{Y}}\left(\boldsymbol{\tau}^{1}(\hat{\boldsymbol{x}}, L, \hat{\boldsymbol{y}}) \boldsymbol{e}_{1}-\boldsymbol{\tau}^{1}(\hat{\boldsymbol{x}},-L, \hat{\boldsymbol{y}}) \boldsymbol{e}_{1}\right) d \hat{\boldsymbol{y}}-2 L \frac{\partial \boldsymbol{\sigma}^{0}}{\partial x_{1}}(0, \hat{\boldsymbol{x}}) \boldsymbol{e}_{1}= \\
& |I| \boldsymbol{d i v}_{x}\left(\left(\boldsymbol{A}-\boldsymbol{A}^{I}\right) \boldsymbol{\gamma}_{x}\left(\boldsymbol{u}^{0}(0, \hat{\boldsymbol{x}})\right)\right)-\boldsymbol{d i v}_{x}\left(\frac{\partial u_{i}^{0}}{\partial x_{j}} \int_{Y} \boldsymbol{T}^{i j}(\boldsymbol{y}) d \boldsymbol{y}\right) .
\end{aligned}
$$

- for $p>0$

$$
\int_{\hat{Y}}\left(\boldsymbol{\tau}^{1}(\hat{\boldsymbol{x}}, L, \hat{\boldsymbol{y}}) \boldsymbol{e}_{1}-\boldsymbol{\tau}^{1}(\hat{\boldsymbol{x}},-L, \hat{\boldsymbol{y}}) \boldsymbol{e}_{1}\right) d \hat{\boldsymbol{y}}=-\int_{Y_{L} \backslash I} \boldsymbol{d i v}_{x} \boldsymbol{\tau}^{0}(\hat{\boldsymbol{x}}, \boldsymbol{y}) d \boldsymbol{y}
$$


and as in the previous case one obtains:

$$
\begin{gathered}
\int_{\hat{Y}}\left(\boldsymbol{\tau}^{1}(\hat{\boldsymbol{x}}, L, \hat{\boldsymbol{y}}) \boldsymbol{e}_{1}-\boldsymbol{\tau}^{1}(\hat{\boldsymbol{x}},-L, \hat{\boldsymbol{y}}) \boldsymbol{e}_{1}\right) d \hat{\boldsymbol{y}}-2 L \frac{\partial \boldsymbol{\sigma}^{0}}{\partial x_{1}}(0, \hat{\boldsymbol{x}}) \boldsymbol{e}_{1}= \\
|I| \boldsymbol{\boldsymbol { d i v } _ { x }}\left(\boldsymbol{A} \boldsymbol{\gamma}_{x}\left(\boldsymbol{u}^{0}(0, \hat{\boldsymbol{x}})\right)\right)-\boldsymbol{d i} \boldsymbol{v}_{x}\left(\frac{\partial u_{i}^{0}}{\partial x_{j}} \int_{Y \backslash I} \boldsymbol{T}^{i j}(\boldsymbol{y}) d \boldsymbol{y}\right)
\end{gathered}
$$

In conclusion, taking $\lim _{L \rightarrow \infty}$ one finds the second transmission condition on $\omega$ :

$$
\left[\boldsymbol{\sigma}^{1} \boldsymbol{e}_{1}\right]_{\omega}= \begin{cases}\boldsymbol{d i v}_{x}\left(\frac{|I|}{|\hat{Y}|}\left(\boldsymbol{A}-\boldsymbol{A}^{I}\right) \boldsymbol{\gamma}_{x}\left(\boldsymbol{u}^{0}(0, \hat{\boldsymbol{x}})\right)-\frac{\partial u_{i}^{0}}{\partial x_{j}}(0, \hat{\boldsymbol{x}}) \frac{1}{|\hat{Y}|} \int_{Y} \boldsymbol{T}^{i j}(\boldsymbol{y}) d \boldsymbol{y}\right) & \text { if } p=0 \\ \boldsymbol{d i v}_{x}\left(\frac{|I|}{|\hat{Y}|} \boldsymbol{A} \boldsymbol{\gamma}_{x}\left(\boldsymbol{u}^{0}(0, \hat{\boldsymbol{x}})\right)-\frac{\partial u_{i}^{0}}{\partial x_{j}}(0, \hat{\boldsymbol{x}}) \frac{1}{|\hat{Y}|} \int_{Y \backslash I} \boldsymbol{T}^{i j}(\boldsymbol{y}) d \boldsymbol{y}\right) & \text { if } p>0\end{cases}
$$

Let us state explicitly that the missing transmission conditions obtained for $p>0$ coincide with the analogous transmission conditions obtained in the case of the holes in Ref. 9.

Remark 4.3. It is worthwhile to remark that the solution $\boldsymbol{u}^{1}$ of (4.21) in general cannot be in $\boldsymbol{H}^{1}(\Omega)$. One only has $\boldsymbol{u}^{1} \in \boldsymbol{Z}$ where $\boldsymbol{Z}:=\left\{\boldsymbol{z} \in \boldsymbol{L}^{2}(\Omega) ; \boldsymbol{d i v} \boldsymbol{A} \boldsymbol{\gamma}\left(\boldsymbol{z}^{ \pm}\right)=\right.$ $\mathbf{0}$ in $\Omega^{ \pm}$, where $\left.\boldsymbol{z}^{ \pm}=\left.\boldsymbol{z}\right|_{\Omega^{ \pm}}\right\}$. Hence the transmission conditions of (4.21) have to be taken in a weak sense adapting the methods of Lions-Magenes Ref. 18.

\section{Numerical validation}

The numerical validation needs at first the computation with a standard finite element method of a reference solution $\boldsymbol{u}_{h}^{\varepsilon}$ on a suitable mesh for a test problem and for decreasing values of $\varepsilon$. We then compare this computed reference solution with the solutions $\boldsymbol{u}_{0, h}^{\text {out }}=\boldsymbol{u}_{h}^{0}$ and $\boldsymbol{u}_{1, h}^{\text {out }}=\boldsymbol{u}_{h}^{0}+\varepsilon \boldsymbol{u}_{h}^{1}$ obtained by the asymptotic approach. The aim will be to verify numerically that these approximations improve when $\varepsilon$ decreases and also for each fixed $\varepsilon$ far from $\omega$, i.e. far from the heterogeneities. For this we test the approximations on the family of domains $\Omega^{\text {out }, \delta}:=\left\{\boldsymbol{x} \in \Omega ;\left|x_{1}\right|>\right.$ $\left.\frac{\delta}{2}\right\}$ varying the parameter $\delta$.

\subsection{Description of the test problem}

Let be $\Omega=(-L, L) \times(-H, H)$ a plane domain containing $\mathcal{N}(\varepsilon)=2 H \varepsilon^{-1}$ identical discs of diameter $\varepsilon D$, arranged periodically (with period $\varepsilon$ ), along the line $\omega$ of equation $x_{1}=0$. Outside the heterogeneities the structure is constituted of an elastic homogeneous isotropic material, characterized by its Young modulus $E$ and Poisson coefficient $\nu$. The elastic homogeneous isotropic material forming the heterogeneities 
is characterized by the Young modulus $E^{\varepsilon, I}=\varepsilon^{p} E^{I}$ and the Poisson coefficient $\nu^{\varepsilon, I}=\nu$. The boundary $\Gamma_{0}$ of the structure is clamped and a density $\boldsymbol{F}$ of linear force is applied on the complementary part $\Gamma_{F}$. There are no body forces (see Fig.8). In order to evaluate the asymptotic model, we choose four values of $\varepsilon: \varepsilon=\frac{2 H}{10}$, $\varepsilon=\frac{2 H}{20}, \varepsilon=\frac{2 H}{40}$ and $\varepsilon=\frac{2 H}{80}$. And for each value of $\varepsilon$ we consider two values of $E^{\varepsilon, I}: E^{\varepsilon, I}=\varepsilon^{0} \frac{E}{2}$ and $E^{\varepsilon, I}=\varepsilon \frac{E}{2}$. Each problem is solved considering the plane stress approximation.

Let us remark that, in order to capture the influence of the heterogeneities, for

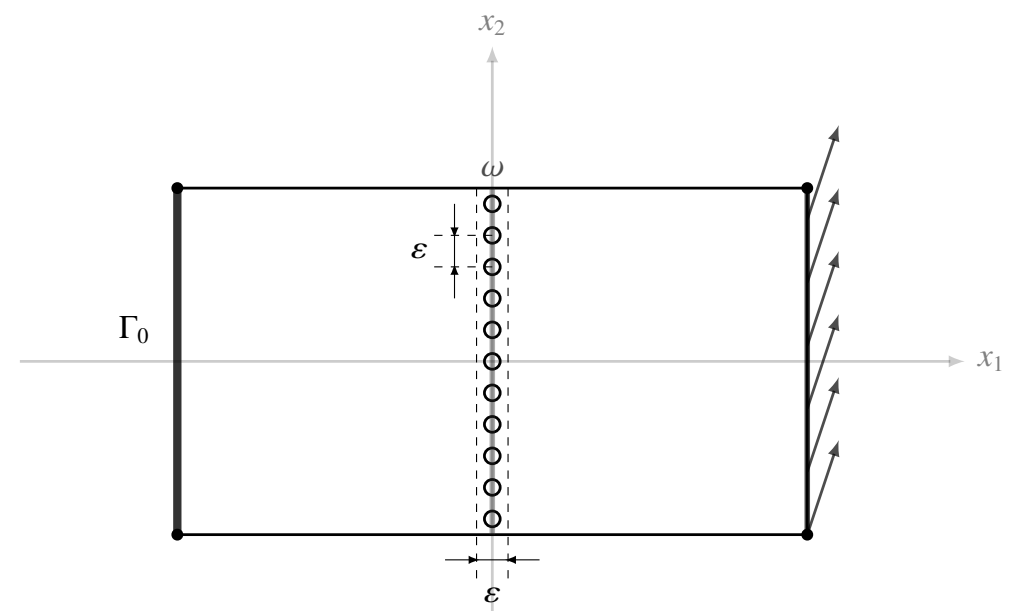

Fig. 8. Test Problem

each value of $\varepsilon$ one has to use a suitably fine mesh so that the computed reference solution $\boldsymbol{u}_{h}^{\varepsilon}$ be precise enough. Since in the finite element computations we have used P2 Lagrange elements the error in the energy norm will be of order $O\left(h^{2}\right)$ (and of order $O\left(h^{3}\right)$ in the $L^{2}$-norm) where as usual $h$ corresponds to the diameter of the largest element, see e.g. Ref. 7. In our numerical simulations we have chosen for all values of $\varepsilon$ the same value of $h$. Let us point out that in practice the mesh will be non uniform since the mesh used near the heterogeneities must have a diameter $<\varepsilon$; this rapidly gives a very stringent condition on the number of the elements of the mesh.

\subsection{Validation of the outer approximations in all $\Omega$}

We compute $\boldsymbol{u}_{h}^{0}$ on the full domain $\Omega$ by a standard finite element method with a uniform mesh of size $h$; for the computation of $\boldsymbol{u}_{h}^{1}$ we use a suitable domain decomposition method (see Ref. 10 for the details). In this way we obtain $\boldsymbol{u}_{0, h}^{\text {out }}$ and $\boldsymbol{u}_{1, h}^{\text {out }}$. These computations are done for $p=0$ and $p=1$. Let us explicitly remark 
that in the comparison with $\boldsymbol{u}_{h}^{\varepsilon}$ the value of $\varepsilon$ plays an important role since when $\varepsilon$ decreases the number of heterogeneities increases.

For $\boldsymbol{u}_{0, h}^{\text {out }}$ the validation is performed by computing the relative error for the energy norm $\left\|\boldsymbol{u}_{h}^{\varepsilon}-\boldsymbol{u}_{0, h}^{\text {out }}\right\|_{\Omega} /\left\|\boldsymbol{u}_{h}^{\varepsilon}\right\|_{\Omega}$ where for a given vector field $\boldsymbol{w}$ the energy norm is defined by:

$$
\|\boldsymbol{w}\|_{\Omega}^{2}:=\int_{\Omega} \boldsymbol{A}^{\varepsilon} \gamma(\boldsymbol{w}) \gamma(\boldsymbol{w}) d \boldsymbol{x} .
$$

As pointed out in Remark $4.3 \boldsymbol{u}_{1} \in \boldsymbol{Z} \subset \boldsymbol{L}^{2}(\Omega)$; hence the validation for $\boldsymbol{u}_{1, h}^{\text {out }}$ is performed by computing the relative error for the $L^{2}$-norm $\left|\boldsymbol{u}_{h}^{\varepsilon}-\boldsymbol{u}_{1, h}^{\text {out }}\right|_{\Omega} /\left|\boldsymbol{u}_{h}^{\varepsilon}\right|_{\Omega}$ where for a given vector field $\boldsymbol{w}$ the $L^{2}$-norm is defined by

$$
|\boldsymbol{w}|_{\Omega}^{2}:=\int_{\Omega} \boldsymbol{w}^{2} d \boldsymbol{x} .
$$

The results obtained for the cases $p=0$ and $p=1$ are given in the following tables.

Table 1. Relative errors for $p=0$.

\begin{tabular}{|c|c|c|c|}
\hline$\varepsilon$ & $\frac{\left\|\boldsymbol{u}_{h}^{\varepsilon}-\boldsymbol{u}_{0, h}^{\text {out }}\right\|_{\Omega}}{\left\|\boldsymbol{u}_{h}^{\varepsilon}\right\|_{\Omega}}$ & $\frac{\left|\boldsymbol{u}_{h}^{\varepsilon}-\boldsymbol{u}_{0, h}^{\text {out }}\right|_{\Omega}}{\left|\boldsymbol{u}_{h}^{\varepsilon}\right|_{\Omega}}$ & $\frac{\left|\boldsymbol{u}_{h}^{\varepsilon}-\boldsymbol{u}_{1, h}^{\text {out }}\right|_{\Omega}}{\left|\boldsymbol{u}_{h}^{\varepsilon}\right|_{\Omega}}$ \\
\hline $1 / 10$ & 0.0581 & 0.00839 & 0.00078 \\
$1 / 20$ & 0.0413 & 0.00421 & 0.00034 \\
$1 / 40$ & 0.0289 & 0.00210 & 0.00015 \\
$1 / 80$ & 0.0205 & 0.00104 & 0.00006 \\
\hline
\end{tabular}

Table 2. Relative errors for $p=1$.

\begin{tabular}{|c|c|c|c|}
\hline$\varepsilon$ & $\frac{\left\|\boldsymbol{u}_{h}^{\varepsilon}-\boldsymbol{u}_{0, h}^{\text {out }}\right\|_{\Omega}}{\left\|\boldsymbol{u}_{h}^{\varepsilon}\right\|_{\Omega}}$ & $\frac{\left|\boldsymbol{u}_{h}^{\varepsilon}-\boldsymbol{u}_{0, h}^{\text {out }}\right|_{\Omega}}{\left|\boldsymbol{u}_{h}^{\varepsilon}\right|_{\Omega}}$ & $\frac{\left|\boldsymbol{u}_{h}^{\varepsilon}-\boldsymbol{u}_{1, h}^{\text {out }}\right|_{\Omega}}{\left|\boldsymbol{u}_{h}^{\varepsilon}\right|_{\Omega}}$ \\
\hline $1 / 10$ & 0.16493 & 0.03603 & 0.00796 \\
$1 / 20$ & 0.12412 & 0.02008 & 0.00215 \\
$1 / 40$ & 0.09057 & 0.01062 & 0.00055 \\
$1 / 80$ & 0.06484 & 0.00542 & 0.00016 \\
\hline
\end{tabular}

\subsection{Validation of the outer approximations far from $\omega$}

The construction of the matched asymptotic expansion method has as Ansatz that the outer approximations are of better quality far from $\omega$. In order to verify this assumption we define the following family of varying outer domains: $\left.\Omega^{\text {out }, \delta}=\Omega \backslash(\delta) \times(-H, H)\right)$. Let us remark that the classical regularity results for elliptic equations imply that $\boldsymbol{u}^{1} \in \boldsymbol{H}^{1}\left(\Omega^{\text {out }, \delta}\right)$; hence for every domain we can 
compute the relative errors $\frac{\left\|\boldsymbol{u}_{h}^{\varepsilon}-\boldsymbol{u}_{p, h}^{\text {out }}\right\|_{\delta}}{\left\|\boldsymbol{u}_{h}^{\varepsilon}\right\|_{\delta}}$ in the energy norms that now are defined as:

$$
\|\boldsymbol{w}\|_{\delta}^{2}:=\int_{\Omega^{\text {out }, \delta}} \boldsymbol{A}^{\varepsilon} \boldsymbol{\gamma}(\boldsymbol{w}) \boldsymbol{\gamma}(\boldsymbol{w}) d \boldsymbol{x} .
$$

The numerical results for decreasing values of $\varepsilon$ and increasing values of $\delta$ are given for $p=0$ in tables 3 and 4 , and for $p=1$ in tables 5 and 6 . Let us explicitly point

Table 3. Relative errors $\frac{\left\|\boldsymbol{u}_{h}^{\varepsilon}-\boldsymbol{u}_{0, h}^{\text {out }}\right\|_{\delta}}{\left\|\boldsymbol{u}_{h}^{\varepsilon}\right\|_{\delta}}$ for $p=0$.

\begin{tabular}{|c|c|c|c|c|}
\hline$\delta$ & $\varepsilon=0.1$ & $\varepsilon=0.05$ & $\varepsilon=0.025$ & $\varepsilon=0.0125$ \\
\hline 0.01 & 0.0499 & 0.03065 & 0.01527 & 0.00508 \\
0.03 & 0.0399 & 0.01480 & 0.00378 & 0.00284 \\
0.06 & 0.0214 & 0.00614 & 0.00205 & 0.00281 \\
0.10 & 0.0135 & 0.00239 & 0.00188 & 0.00280 \\
0.15 & 0.00547 & 0.00182 & 0.00178 & 0.00282 \\
0.20 & 0.00335 & 0.00155 & 0.00173 & 0.00284 \\
0.25 & 0.00271 & 0.00139 & 0.00172 & 0.00286 \\
0.30 & 0.00243 & 0.00128 & 0.00171 & 0.00289 \\
\hline
\end{tabular}

Table 4. Relative errors $\frac{\left\|\boldsymbol{u}_{h}^{\varepsilon}-\boldsymbol{u}_{1, h}^{o u t}\right\|_{\delta}}{\left\|\boldsymbol{u}_{h}^{\varepsilon}\right\|_{\delta}}$ for $p=0$.

\begin{tabular}{|c|c|c|c|c|}
\hline$\delta$ & $\varepsilon=0.1$ & $\varepsilon=0.05$ & $\varepsilon=0.025$ & $\varepsilon=0.0125$ \\
\hline 0.01 & 0.0499 & 0.03062 & 0.01523 & 0.00505 \\
0.03 & 0.0398 & 0.01467 & 0.00364 & 0.00279 \\
0.06 & 0.0212 & 0.00590 & 0.00186 & 0.00278 \\
0.10 & 0.0133 & 0.00193 & 0.00174 & 0.00278 \\
0.15 & 0.00500 & 0.00139 & 0.00168 & 0.00280 \\
0.20 & 0.00274 & 0.00118 & 0.00166 & 0.00282 \\
0.25 & 0.00207 & 0.00105 & 0.00166 & 0.00285 \\
0.30 & 0.00184 & 0.00098 & 0.00166 & 0.00288 \\
\hline
\end{tabular}

Table 5. Relative errors $\frac{\left\|\boldsymbol{u}_{h}^{\varepsilon}-\boldsymbol{u}_{0, h}^{\text {out }}\right\|_{\delta}}{\left\|\boldsymbol{u}_{h}^{\varepsilon}\right\|_{\delta}}$ for $p=1$.

\begin{tabular}{|c|c|c|c|c|}
\hline$\delta$ & $\varepsilon=0.1$ & $\varepsilon=0.05$ & $\varepsilon=0.025$ & $\varepsilon=0.0125$ \\
\hline 0.01 & 0.14870 & 0.10510 & 0.06074 & 0.02165 \\
0.03 & 0.12769 & 0.06880 & 0.01676 & 0.00472 \\
0.06 & 0.09096 & 0.02833 & 0.00629 & 0.00419 \\
0.10 & 0.05783 & 0.00976 & 0.00512 & 0.00378 \\
0.15 & 0.02224 & 0.00712 & 0.00428 & 0.00349 \\
0.20 & 0.01212 & 0.00598 & 0.00374 & 0.00334 \\
0.25 & 0.00894 & 0.00524 & 0.00339 & 0.00326 \\
0.30 & 0.00764 & 0.00470 & 0.00313 & 0.00322 \\
\hline
\end{tabular}


Table 6. Relative errors $\frac{\left\|\boldsymbol{u}_{h}^{\varepsilon}-\boldsymbol{u}_{1, h}^{\text {out }}\right\|_{\delta}}{\left\|\boldsymbol{u}_{h}^{\varepsilon}\right\|_{\delta}}$ for $p=1$.

\begin{tabular}{|c|c|c|c|c|}
\hline$\delta$ & $\varepsilon=0.1$ & $\varepsilon=0.05$ & $\varepsilon=0.025$ & $\varepsilon=0.0125$ \\
\hline 0.01 & 0.14937 & 0.10484 & 0.06040 & 0.02131 \\
0.03 & 0.12787 & 0.06815 & 0.01563 & 0.00329 \\
0.06 & 0.09075 & 0.02712 & 0.00353 & 0.00304 \\
0.10 & 0.05761 & 0.00700 & 0.00275 & 0.00293 \\
0.15 & 0.02179 & 0.00431 & 0.00232 & 0.00288 \\
0.20 & 0.01140 & 0.00342 & 0.00208 & 0.00287 \\
0.25 & 0.00808 & 0.00286 & 0.00193 & 0.00288 \\
0.30 & 0.00674 & 0.00246 & 0.00185 & 0.00290 \\
\hline
\end{tabular}

out that the previous numerical tests are in good agreement with the Ansatz.

\section{Final comments}

1. The numerical validation of the matching asymptotic method suggests that one might give a more rigorous proof, eventually using variational convergence methods at least for $\boldsymbol{u}_{0}^{\text {out }}$. Indeed e.g. in Ref. $4,5,6,12, \ldots$ using variational convergence methods has been studied the situation of a full heterogeneous $\varepsilon$-thin layer whose elastic coefficients $\boldsymbol{A}^{\varepsilon, L}$ satisfy:

$$
\boldsymbol{A}^{\varepsilon, L}=\varepsilon^{p} \boldsymbol{A}^{L}, \boldsymbol{A}^{L} \simeq \boldsymbol{A},
$$

where $p$ takes positive or negative values. In all the situations considered in these papers the influence of the layer appears in the zero order approximation whereas Theorem 4.1 states that there is no influence of the heterogeneities at zero order.

2. As has been observed in Remark 4.3 the non-homogeneous transmission problem (4.21) is non-classical since the data are in Sobolev spaces with highly negative exponents. For this reason it is natural to search the solution $\boldsymbol{u}^{1} \in \boldsymbol{Z}$; however the corresponding theory has to be carefully developed (following the methods used in Ref. 18) when $\omega$ is smooth and $\partial \omega \cap \partial \Omega=\emptyset$. Also when the data are regular some singularities are present in $\partial \omega \cap \partial \Omega$ as can also be seen in the numerical experiments, see Ref. 10 and see Ref. 13 for a general study of these singularities.

3. One could consider a more general situation with many parameters: for instance one can study what happens if the diameter of the heterogeneities is of the type $\varepsilon^{m} d$ with $m>1$.

4. In a forthcoming paper Ref. 11 we study some situations of strong heterogeneities.

\section{Acknowledgment}

This work was partially supported by the French Agence Nationale de la Recherche (ANR) under Grant Epsilon (BLAN08-2 312370) (Domain decomposition and multi-scale computations of singularities in mechanical structures); it is a natural continuation of the Thesis of SH carried out at I3M and INRIA and supported by Grant Epsilon. 


\section{References}

1. R. Abdelmoula, J. J. Marigo, The effective behavior of a fiber bridged crack, J. Mech. Phys. Solids 48 (2000) 2419 - 2444.

2. M. Bellieud, G. Geymonat, F. Krasucki, G. Michaille. In preparation.

3. A. Bensoussan, J.L. Lions, G. Papanicolaou, Asymptotic analysis for periodic structures, ( North Holland, Amsterdam, 1987).

4. A. L. Bessoud, F. Krasucki and G. Michaille, Multi-materials with strong interface: variational modelings, Asymptotic Analysis 61, (2009) 1-19.

5. A.L. Bessoud, F. Krasucki and M. Serpilli, Plate-like and shell-like inclusions with high rigidity, C. R. Acad. Sci. Paris, Ser. I, 346 (2008) 697-702.

6. A. L. Bessoud, F. Krasucki and M. Serpilli, Asymptotic analysis of shell-like inclusions with high rigidity, Journal of Elasticity 103 (2011) 153-172, DOI: 10.1007/s10659-0109278-1.

7. P.G. Ciarlet, The Finite element Method for Elliptic Problems, (SIAM, 2002).

8. M. David, J.J. Marigo and C. Pideri, Homogenized Interface Model Describing Inhomogeneities Localized on a Surface, Journal of Elasticity 109 (2012) 153-187, DOI: 10.1007/s10659-012-9374-5.

9. G. Geymonat, S. Hendili, F. Krasucki and M. Vidrascu, The matched asymptotic expansion for the computation of the effective behavior of an elastic structure with a thin layer of holes, Journal for Multiscale Computational Engineering 9 (2011) 529542.

10. G. Geymonat, S. Hendili, F. Krasucki and M. Vidrascu, Numerical validation of an Homogenized Interface Model, submitted

11. G. Geymonat, S. Hendili, F. Krasucki and M. Vidrascu, In preparation.

12. G. Geymonat, F. Krasucki and S. Lenci, Bonded joint with a soft thin adhesive, Mathematics and Mechanics of Solids 4 (1999) 201-225.

13. P. Grisvard, Singularities in Boundary Values Problems, Masson-Springer Verlag, 1992 ,

14. M.E. Gurtin, The Linear Theory of Elasticity, in Handbuch der Physik Vol. VI a/2, (S. Flügge and C. Truesdell, Editors), pp. 1-295, (Springer-Verlag, 1972).

15. A. M. Il'in, Matching of asymptotic expansions of solutions of boundary value problems, Translations of Mathematical Monographs, 102, (American Mathematical Society, Providence, RI, 1992)

16. A.M. Il'in, A.R. Danilin and S.V. Zakharov Application of the concordance method of asymptotic expansion to solve boundary-value problems, Jour. Mathematical Sciences, 125 (2005) 610-657

17. F. Krasucki and S. Lenci, Analysis of interfaces of variable stiffness, Intern. J. of Solids and Structures, 37 (2000) 3619-3632.

18. J.L. Lions and E. Magenes, Problèmes aux Limites non Homogènes et Applications, Vol1, (Dunod, Paris 1968).

19. J.J. Marigo and C. Pideri,The effective behavior of elastic bodies containing microcracks or microholes localized on a surface, International Journal of Damage Mechanics 20 (2011) 1130-1150.

20. M.M. Mehrabadi and S.C. Cowin, Eigentensors of linear anistropic elastic materials, Quarterly Journal of Mechanics and Applied Mathematics 43 (1990) 15-41.

21. G. Nguetseng and E. Sanchez-Palencia, Stress concentration for defects distributed near a surface, P. Ladevèze (ed.) : Local Effects in the Analysis of Structures, (Elsevier, Amsterdam, 1986) 55-74.

22. J. Rychlewski, On Hookes Law, Journal of Applied Mathematics and Mechanics, 48 (1984) 303-314. 
23. J. Sanchez Hubert and E. Sanchez Palencia, Vibration and coupling of continuous systems. Asymptotic Methods, (Springer Verlag, 1989).

24. E. Sanchez Palencia, Non-homogeneous media and vibration theory, (Springer Verlag, Berlin, 1980).

25. M. Van Dyke, Perturbation Methods in Fluid Mechanics, (Academic Press, New York, 1964) (Annotated Edition, The Parabolic Press, Stanford, 1975). 